\title{
Orthogonal Basic Hypergeometric Laurent Polynomials ${ }^{\star}$
}

Mourad E.H. ISMAIL $\dagger$ and Dennis STANTON

$\dagger$ Department of Mathematics, University of Central Florida, Orlando, FL 32816, USA

E-mail: mourad.eh.ismail@gmail.com

$\ddagger$ Department of Mathematics, King Saud University, Riyadh, Saudi Arabia

$\S$ School of Mathematics, University of Minnesota, Minneapolis, MN 55455, USA

E-mail: stanton@math.umn.edu

Received August 04, 2012, in final form November 28, 2012; Published online December 01, 2012 http://dx.doi.org/10.3842/SIGMA.2012.092

Abstract. The Askey-Wilson polynomials are orthogonal polynomials in $x=\cos \theta$, which are given as a terminating ${ }_{4} \phi_{3}$ basic hypergeometric series. The non-symmetric AskeyWilson polynomials are Laurent polynomials in $z=e^{i \theta}$, which are given as a sum of two terminating ${ }_{4} \phi_{3}$ 's. They satisfy a biorthogonality relation. In this paper new orthogonality relations for single ${ }_{4} \phi_{3}$ 's which are Laurent polynomials in $z$ are given, which imply the non-symmetric Askey-Wilson biorthogonality. These results include discrete orthogonality relations. They can be considered as a classical analytic study of the results for non-symmetric Askey-Wilson polynomials which were previously obtained by affine Hecke algebra techniques.

Key words: Askey-Wilson polynomials; orthogonality

2010 Mathematics Subject Classification: 33D45

\section{Introduction}

The Askey-Wilson polynomials $p_{n}(x ; \mathbf{t} \mid q)$ are polynomials in $x=\cos \theta$, and depend upon parameters $q, t_{1}, t_{2}, t_{3}$, and $t_{4}$. They may be defined by the terminating basic hypergeometric series [1], [8, $\S 15.2]$

$$
p_{n}(x ; \mathbf{t} \mid q)=t_{1}^{-n}\left(t_{1} t_{2}, t_{1} t_{3}, t_{1} t_{4} ; q\right)_{n}{ }_{4} \phi_{3}\left(\begin{array}{c}
q^{-n}, t_{1} t_{2} t_{3} t_{4} q^{n-1}, t_{1} e^{i \theta}, t_{1} e^{-i \theta} \\
t_{1} t_{2}, t_{1} t_{3}, t_{1} t_{4}
\end{array} \mid q, q\right) .
$$

They are orthogonal polynomials in $x$, and have a known orthogonality relation (see (2.1)).

Clearly the Askey-Wilson polynomials are also Laurent polynomials in $z=e^{i \theta}$, since $2 x=$ $z+1 / z$. One may ask if there is a natural basis for Laurent polynomials in $z$ which also satisfy orthogonality relations. The non-symmetric Askey-Wilson polynomials [16, 17] are Laurent polynomials, which are biorthogonal with respect to a modified Askey-Wilson weight, are one answer to this question. They may be explicitly given as a sum of two terminating basic hypergeometric series.

The purpose of this paper is to use classical analytic methods to study the orthogonality relations for single ${ }_{4} \phi_{3}$ 's which imply the non-symmetric Askey-Wilson polynomial biorthogonality. The main orthogonality results are Theorems 3.1, 4.1, 4.4, and 4.5. We show that special function techniques, explicit summations and self-adjoint operators, may be applied to derive

${ }^{\star}$ This paper is a contribution to the Special Issue "Superintegrability, Exact Solvability, and Special Functions". The full collection is available at http://www.emis.de/journals/SIGMA/SESSF2012.html 
these orthogonalities. We explicitly find three and four term relations for Laurent polynomials in Section 4. As a corollary of our computations we rederive the $L^{2}$-norm of the non-symmetric Askey-Wilson polynomials in Section 6, and reprove the creation type formulas for the Laurent polynomials in Section 5. Asymptotics are given in Theorem 9.1, and Racah orthogonality in Theorem 10.2. This work may be considered as a classical study of the one variable case in Cherednik [6], Macdonald [16], carefully studied by Noumi and Stokman [17], who considered many of these results from the point of view of affine Hecke algebras.

There are different theories of orthogonal polynomials, biorthogonal polynomials and rational functions which originate from different types of continued fractions. The $J$-fractions lead to orthogonal polynomials on the line while the $P C$ fractions lead to orthogonal polynomials on the unit circle $[8,11,14,15]$. The theory of $T$-fractions leads to orthogonal Laurent polynomials and is explained in the books $[14,15]$, and the excellent survey article [5]. Ismail and Masson [9] introduced the more general $R_{\mathrm{I}}$ and $R_{\mathrm{II}}$ fractions which naturally lead to biorthogonal orthogonal rational functions. A. Zhedanov [21] pointed out the connection of the latter continued fractions and the generalized eigenvalue problem [19]. For applications of the $R_{\mathrm{I}}$ and $R_{\mathrm{II}}$ fractions to integrable systems, see [18]. There is a more abstract approach to biorthogonality presented in Brezinski's monograph [4] with many applications to numerical analysis and computational mathematics.

\section{Notation}

Let $V_{n}$ be the $(2 n+1)$-dimensional real vector space spanned by the Laurent polynomials $\left\{z^{-n}, z^{1-n}, \ldots, z^{n-1}, z^{n}\right\}$. Most of this section is devoted to notation for specific spanning sets in $V_{n}$. These sets have orthogonality relations given in later sections, we define the appropriate measures here.

We assume throughout the paper that $x=\cos \theta$ and $z=e^{i \theta}$, and $\mathbf{t}$ stands for the vector $\left(t_{1}, t_{2}, t_{3}, t_{4}\right)$. We shall always assume that $0<q<1$ and each $t_{i}$ is real with $\left|t_{i}\right|<1$.

The weight function for the Askey-Wilson polynomials is given by

$$
w_{\mathrm{aw}}(x ; \mathbf{t} \mid q)=\frac{\left(q, e^{2 i \theta}, e^{-2 i \theta} ; q\right)_{\infty}}{\prod_{j=1}^{4}\left(t_{j} e^{i \theta}, t_{j} e^{-i \theta} ; q\right)_{\infty}} .
$$

The Askey-Wilson orthogonality [8, (15.2.4)] is

$$
\begin{aligned}
& \frac{1}{2 \pi} \int_{-1}^{1} p_{m}(x ; \mathbf{t} \mid q) p_{n}(x ; \mathbf{t} \mid q) w_{\mathrm{aw}}(x ; \mathbf{t} \mid q) \frac{d x}{\sqrt{1-x^{2}}} \\
& \quad=\prod_{1 \leq j<k \leq 4}\left(t_{j} t_{k} ; q\right)_{n} \frac{\left(q, t_{1} t_{2} t_{3} t_{4} q^{n-1} ; q\right)_{n}}{\left(t_{1} t_{2} t_{3} t_{4} ; q\right)_{2 n}} \mu(\mathbf{t} \mid q) \delta_{m, n},
\end{aligned}
$$

while the Askey-Wilson integral evaluation is $[3,8]$

$$
\frac{1}{2 \pi} \int_{0}^{\pi} \frac{\left(q, e^{2 i \theta}, e^{-2 i \theta} ; q\right)_{\infty}}{\prod_{j=1}^{4}\left(t_{j} e^{i \theta}, t_{j} e^{-i \theta} ; q\right)_{\infty}} d \theta=\frac{\left(t_{1} t_{2} t_{3} t_{4} ; q\right)_{\infty}}{\prod_{1 \leq j<k \leq 4}\left(t_{j} t_{k} ; q\right)_{\infty}}=\mu(\mathbf{t} \mid q)
$$

The orthogonality relations for Laurent polynomials in $V_{n}$ uses a small variation of the AskeyWilson weight, which is given by

$$
w_{\text {cher }}(z ; \mathbf{t} \mid q)=w_{\text {aw }}(x ; \mathbf{t} \mid q) \frac{\left(1-t_{1} / z\right)\left(1-t_{2} / z\right)}{1-z^{-2}}=\frac{\left(q, z^{2}, q z^{-2} ; q\right)_{\infty}}{\prod_{j=1}^{4}\left(t_{j} z ; q\right)_{\infty} \prod_{k=3}^{4}\left(t_{k} / z, q t_{k-2} / z ; q\right)_{\infty}} .
$$


Even though this weight is not positive on the unit circle, and does not lead to a positive definite quadratic form, we will define a bilinear form using $w_{\text {cher }}(z ; \mathbf{t} \mid q)$, and find orthogonal bases for $V_{n}$ in Sections 3 and 4.

The orthogonality relations will involve Laurent polynomials in $z$ which may be defined in terms of ${ }_{4} \phi_{3}$ functions. We shall need four of these functions: $R_{n}, S_{n}, T_{n}$, and $U_{n}$.

The first polynomial $R_{n}(z, \mathbf{t} \mid q)$ is an unscaled version of the Askey-Wilson polynomials

$$
R_{n}(z ; \mathbf{t} \mid q)={ }_{4} \phi_{3}\left(\begin{array}{c}
q^{-n}, t_{1} t_{2} t_{3} t_{4} q^{n-1}, t_{1} z, t_{1} / z \\
t_{1} t_{2}, t_{1} t_{3}, t_{1} t_{4}
\end{array} \mid q, q\right) .
$$

Put $S_{0}(z)=0$, and for $n \geq 1$,

$$
\begin{aligned}
& S_{n}(z ; \mathbf{t} \mid q)=z\left(1-t_{3} / z\right)\left(1-t_{4} / z\right){ }_{4} \phi_{3}\left(\begin{array}{c}
q^{1-n}, t_{1} t_{2} t_{3} t_{4} q^{n}, t_{1} z, q t_{1} / z \\
q t_{1} t_{2}, q t_{1} t_{3}, q t_{1} t_{4}
\end{array} \mid q, q\right), \\
& T_{n}(z ; \mathbf{t} \mid q)={ }_{4} \phi_{3}\left(\begin{array}{c}
q^{-n}, t_{1} t_{2} t_{3} t_{4} q^{n-1}, t_{1} z, q t_{1} / z \\
q t_{1} t_{2}, t_{1} t_{3}, t_{1} t_{4}
\end{array} \mid q, q\right)
\end{aligned}
$$

and $U_{0}(z)=0$, and for $n \geq 1$,

$$
U_{n}(z ; \mathbf{t} \mid q)=\frac{1}{z}\left(1-t_{1} z\right)\left(1-t_{2} z\right) R_{n-1}\left(z ; q t_{1}, q t_{2}, t_{3}, t_{4} \mid q\right) .
$$

The above representation (2.5) expresses $U_{n}$ as a multiple of a scaled $R_{n}$. The $S_{n}$ and $T_{n}$ in (2.3) and (2.4) can also written in terms of scaled $R_{n}$ 's as follows

$$
\begin{aligned}
& S_{n}(z ; \mathbf{t} \mid q)=z\left(1-t_{3} / z\right)\left(1-t_{4} / z\right) T_{n-1}\left(z ; t_{1}, t_{2}, q t_{3}, q t_{4}\right), \\
& T_{n}(z ; \mathbf{t} \mid q)=R_{n}\left(q^{-1 / 2} z ; q^{1 / 2} t_{1}, q^{1 / 2} t_{2}, q^{-1 / 2} t_{3}, q^{-1 / 2} t_{4}\right) .
\end{aligned}
$$

In other words

$$
S_{n}(z ; \mathbf{t} \mid q)=z\left(1-t_{3} / z\right)\left(1-t_{4} / z\right) R_{n-1}\left(q^{-1 / 2} z ; q^{1 / 2} t_{1}, q^{1 / 2} t_{2}, q^{1 / 2} t_{3}, q^{1 / 2} t_{4}\right) .
$$

Clearly

$$
\left\{R_{0}, \ldots, R_{n}, S_{1}, \ldots, S_{n}, T_{0}, \ldots, T_{n}, U_{1}, \ldots, U_{n}\right\} \subset V_{n},
$$

but this set could not form a basis for $V_{n}$ for $n \geq 0$. There are very specific dependencies amongst these Laurent polynomials which follow from contiguous relations. It follows from Proposition 2.1 that for $n \geq 1$ the subspace $W_{n}=\operatorname{span}\left\{R_{n}, S_{n}, T_{n}, U_{n}\right\}$ of $V_{n}$ is 2-dimensional. Any two of these four Laurent polynomials are linearly independent.

We need some contiguous relations for balanced ${ }_{4} \phi_{3}$ 's. Let

$$
\phi(a, b, c, d ; e, f, g)={ }_{4} \phi_{3}\left(\begin{array}{c}
a, b, c, d \\
e, f, g
\end{array} \mid q, q\right)
$$

denote ${ }_{4} \phi_{3}$ which is terminating and balanced. By $\phi_{+}$we mean change $a, b, \ldots, g$ by $a q, b q$, $\ldots, g q$, respectively, so $\phi_{+}$is no longer balanced. When a parameter $\alpha$ is changed to $\alpha q^{ \pm 1}$ we write $\phi(\alpha \pm)$. Wilson [20] proved contiguous relations which imply

$$
\begin{aligned}
& \phi(a+, e+)-\phi=\frac{q(a-e)(1-b)(1-c)(1-d)}{(1-e)(1-e q)(1-f)(1-g)} \phi_{+}(e+), \\
& \frac{a(1-f / a)(1-g / a)}{(1-f)(1-g)} \phi_{+}(a-)+\frac{(b-e)(1-c / e)}{(1-b)(1-c)} \phi(d+, e+)=\frac{(1-e)(1-b c / e)}{(1-b)(1-c)} \phi .
\end{aligned}
$$


Proposition 2.1. The following connection relations hold for $n \geq 1$,

$$
\begin{aligned}
& T_{n}(z ; \mathbf{t} \mid q)-R_{n}(z ; \mathbf{t} \mid q)=\frac{q t_{1}\left(1-q^{-n}\right)\left(1-t_{1} t_{2} t_{3} t_{4} q^{n-1}\right)}{\left(1-t_{1} t_{2}\right)\left(1-q t_{1} t_{2}\right)\left(1-t_{1} t_{3}\right)\left(1-t_{1} t_{4}\right)} U_{n}(z ; \mathbf{t} \mid q), \\
& \left(1-t_{1} t_{3}\right)\left(1-t_{1} t_{4}\right) R_{n}(z ; \mathbf{t} \mid q)-t_{1} S_{n}(z ; \mathbf{t} \mid q)=\frac{t_{1}\left(1-t_{1} t_{2} q^{n}\right)\left(1-t_{3} t_{4} q^{n-1}\right)}{q^{n-1}\left(1-t_{1} t_{2}\right)\left(1-q t_{1} t_{2}\right)} U_{n}(z ; \mathbf{t} \mid q), \\
& \frac{t_{1}}{\left(1-t_{1} t_{3}\right)\left(1-t_{1} t_{4}\right)} S_{n}(z ; \mathbf{t} \mid q)-\frac{\left(1-q^{n} t_{1} t_{2}\right)\left(1-q^{n-1} t_{3} t_{4}\right)}{\left(1-q^{n}\right)\left(1-t_{1} t_{2} t_{3} t_{4} q^{n-1}\right)} T_{n}(z ; \mathbf{t} \mid q) \\
& \quad+\frac{q^{n}\left(1-t_{1} t_{2}\right)\left(1-t_{3} t_{4} / q\right)}{\left(1-q^{n}\right)\left(1-t_{1} t_{2} t_{3} t_{4} q^{n-1}\right)} R_{n}(z ; \mathbf{t} \mid q)=0 .
\end{aligned}
$$

Proof. The first statement follows from (2.6) with $a=t_{1} / z, b=q^{-n}, c=t_{1} t_{2} t_{3} t_{4} q^{n-1}, d=t_{1} z$, $e=t_{1} t_{2}, f=t_{1} t_{3}$, and $g=t_{1} t_{4}$. The third statement follows from from (2.7) with $a=t_{1} z$, $b=q^{-n}, c=t_{1} t_{2} t_{3} t_{4} q^{n-1}, d=t_{1} / z, e=t_{1} t_{2}, f=t_{1} t_{3}$, and $g=t_{1} t_{4}$. The second statement follows from the other two.

We will also use the Sears transformation [7, (III.15)],

$$
{ }_{4} \phi_{3}\left(\begin{array}{c}
q^{-n}, A, B, C \\
D, E, F
\end{array} \mid q, q\right)=\frac{(E / A, F / A ; q)_{n}}{(E, F ; q)_{n}} A^{n}{ }_{4} \phi_{3}\left(\begin{array}{c}
q^{-n}, A, D / B, D / C \\
D, A q^{1-n} / E, A q^{1-n} / F
\end{array} \mid q, q\right),
$$

where $D E F=A B C q^{1-n}$. The Sears transformation (2.8) applied to the $R_{n}$ and $T_{n}$ functions give

$$
R_{n}\left(z ; t_{1}, t_{2}, t_{3}, t_{4} \mid q\right)=\frac{t_{1}^{n}\left(t_{2} t_{3}, t_{3} t_{4} ; q\right)_{n}}{t_{3}^{n}\left(t_{1} t_{2}, t_{1} t_{4} ; q\right)_{n}} R_{n}\left(z ; t_{3}, t_{2}, t_{1}, t_{4} \mid q\right)
$$

and

$$
T_{n}\left(z ; t_{1}, t_{2}, t_{3}, t_{4} \mid q\right)=\frac{t_{1}^{n}\left(t_{2} t_{3}, t_{2} t_{4} ; q\right)_{n}}{t_{2}^{n}\left(t_{1} t_{3}, t_{1} t_{4} ; q\right)_{n}} T_{n}\left(z ; t_{2}, t_{1}, t_{3}, t_{4} \mid q\right)
$$

One may also easily see from the definition of $R_{n}$ and $S_{n}$ that

$$
R_{n}(1 / z ; 1 / \mathbf{t} \mid 1 / q)=R_{n}(z ; \mathbf{t} \mid q), \quad S_{n}(1 / z ; 1 / \mathbf{t} \mid 1 / q)=S_{n}(z ; \mathbf{t} \mid q) / t_{3} t_{4} .
$$

\section{Orthogonality relations}

The main result of this section is Theorem 3.1.

For Laurent polynomials $f(z)$ and $g(z)$ define a bilinear form by

$$
\langle f, g\rangle_{\mathrm{cher}}=\frac{1}{2 \pi i} \oint_{|z|=1} f(z) g(z) w_{\mathrm{cher}}(z ; \mathbf{t} \mid q) \frac{d z}{z} .
$$

Theorem 3.1. The set $\left\{R_{0}, \ldots, R_{n}, U_{1}, \ldots, U_{n}\right\}$ is an orthogonal basis for $V_{n}$ with respect to the symmetric bilinear form $\langle,\rangle_{\text {cher. }}$. Moreover, $\left\langle R_{m}, R_{m}\right\rangle_{\text {cher }}$ and $\left\langle U_{m}, U_{m}\right\rangle_{\text {cher }}$ are non-zero.

The proof of Theorem 3.1 will be accomplished in three stages. We get $R_{n}-R_{m}$ orthogonality for "free". We establish in Propositions 3.2 and 3.4 orthogonalities between $R_{n}$ and $T_{m}$, which changes into $R_{n}-U_{m}$ and $U_{n}-U_{m}$ orthogonality.

First we establish $R_{n}-R_{m}$ orthogonality from Askey-Wilson orthogonality. It is a restatement of (2.1), which was given in [17, Lemma 6.4]. 
Proposition 3.2. The orthogonality relation for the $R_{n}$ functions is

$$
\left\langle R_{m}, R_{n}\right\rangle_{\text {cher }}=\frac{\left(1-t_{1} t_{2}\right) t_{1}^{2 n}\left(q, t_{2} t_{3}, t_{2} t_{4}, t_{3} t_{4}, t_{1} t_{2} t_{3} t_{4} q^{n-1} ; q\right)_{n}}{\left(t_{1} t_{2}, t_{1} t_{3}, t_{1} t_{4} ; q\right)_{n}\left(t_{1} t_{2} t_{3} t_{4} ; q\right)_{2 n}} \mu(\mathbf{t} \mid q) \delta_{m, n} .
$$

Proof. Rewrite the left-hand side of Proposition 3.2 as

$$
\begin{aligned}
& \frac{1}{2 \pi} \int_{-\pi}^{\pi} R_{m}\left(e^{i \theta} ; \mathbf{t} \mid q\right) R_{n}\left(e^{i \theta} ; \mathbf{t} \mid q\right) w_{\mathrm{aw}}(\cos \theta ; \mathbf{t} \mid q) \frac{e^{i \theta}-\left(t_{1}+t_{2}\right)+t_{1} t_{2} e^{-i \theta}}{2 \sin \theta} d \theta \\
& \quad=\frac{1-t_{1} t_{2}}{2 \pi} \int_{0}^{\pi} R_{m}\left(e^{i \theta} ; \mathbf{t} \mid q\right) R_{n}\left(e^{i \theta} ; \mathbf{t} \mid q\right) w_{\mathrm{aw}}(\cos \theta ; \mathbf{t} \mid q) d \theta
\end{aligned}
$$

since $R_{n}\left(e^{i \theta} ; \mathbf{t} \mid q\right)$ and $w_{\text {aw }}$ are even functions of $\theta$.

To establish the $U_{n}-R_{m}$ orthogonality we first give an integral evaluation for "gluing" a specific Laurent polynomial onto the measure.

Lemma 3.3. For non-negative integers $k$ and $j$ we have

$$
\begin{aligned}
& \frac{1}{2 \pi i} \oint_{|z|=1}\left(t_{1} z, q t_{1} / z ; q\right)_{j}\left(t_{3} z, t_{3} / z ; q\right)_{k} w_{\text {cher }}(z ; \mathbf{t} \mid q) \frac{d z}{z} \\
& \quad=\frac{\left(t_{1} t_{2} t_{3} t_{4} q^{j+k} ; q\right)_{\infty}}{\left(t_{1} t_{2} q^{j+1}, t_{1} t_{3} q^{j+k}, t_{1} t_{4} q^{j}, t_{2} t_{3} q^{k}, t_{2} t_{4}, t_{3} t_{4} q^{k} ; q\right)_{\infty}} .
\end{aligned}
$$

Proof. The integral is

$$
\frac{1}{2 \pi i} \oint_{|z|=1} \frac{\left(q, z^{2}, z^{-2} ; q\right)_{\infty}}{\left(t_{1} q^{j} z, t_{2} z, t_{3} q^{k} z, t_{4} z, q^{j} t_{1} / z, t_{2} / z, q^{k} t_{3} / z, t_{4} / z ; q\right)_{\infty}} \frac{\left(1-t_{2} / z\right)\left(1-q^{j} t_{1} / z\right)}{\left(1-z^{-2}\right)} \frac{d z}{z} .
$$

This time the rational function in $z=e^{i \theta}$ in the integrand is

$$
\frac{e^{i \theta}-\left(q^{j} t_{1}+t_{2}\right)+t_{1} t_{2} q^{j} e^{-i \theta}}{2 i \sin (\theta)} .
$$

The remaining part of the integrand is symmetric in $\theta$, so only the odd part of the numerator in (3.1) contributes. The Askey-Wilson integral evaluation gives the result

$$
\begin{gathered}
=\frac{1}{2}\left(1-t_{1} t_{2} q^{j}\right) \frac{1}{2 \pi} \int_{-\pi}^{\pi} w_{\text {aw }}\left(\cos \theta ; q^{j} t_{1}, t_{2}, q^{k} t_{3}, t_{4} \mid q\right) d \theta \\
=\frac{\left(t_{1} t_{2} t_{3} t_{4} q^{j+k} ; q\right)_{\infty}}{\left(t_{1} t_{2} q^{j+1}, t_{1} t_{3} q^{j+k}, t_{1} t_{4} q^{j}, t_{2} t_{3} q^{k}, t_{2} t_{4}, t_{3} t_{4} q^{k} ; q\right)_{\infty}} .
\end{gathered}
$$

Proposition 3.4. We have the orthogonality relation

$$
\left\langle R_{m}, T_{n}\right\rangle_{\mathrm{cher}}=\left\langle R_{m}, R_{n}\right\rangle_{\mathrm{cher}} \delta_{m, n} .
$$

Proof. The proof is done in three stages: $m<n, m=n$, and $m>n$.

Let $I_{j, k}$ be the integral in Lemma 3.3. Using the explicit definition of $T_{n}(z ; \mathbf{t} \mid q)$ we have

$$
\begin{aligned}
& \frac{1}{2 \pi i} \oint_{|z|=1}\left(t_{3} z, t_{3} / z ; q\right)_{k} T_{n}(z ; \mathbf{t} \mid q) w_{\text {cher }}(z ; \mathbf{t} \mid q) \frac{d z}{z}=\sum_{j=0}^{n} \frac{\left(q^{-n}, t_{1} t_{2} t_{3} t_{4} q^{n-1} ; q\right)_{j}}{\left(q, q t_{1} t_{2}, t_{1} t_{3}, t_{1} t_{4} ; q\right)_{j}} q^{j} I_{j, k} \\
& \quad=\frac{\left(t_{1} t_{2} t_{3} t_{4} q^{k} ; q\right)_{\infty}}{\left(q, q t_{1} t_{2}, q^{k} t_{1} t_{3}, t_{1} t_{4}, q^{k} t_{2} t_{3}, t_{2} t_{4}, q^{k} t_{3} t_{4} ; q\right)_{\infty}}{ }_{\infty} \phi_{2}\left(\begin{array}{c}
q^{-n}, t_{1} t_{2} t_{3} t_{4} q^{n-1}, q^{k} t_{1} t_{3} \\
t_{1} t_{3}, t_{1} t_{2} t_{3} t_{4} q^{k}
\end{array} \mid q, q\right) .
\end{aligned}
$$


From the $q$-Pfaff-Saalschütz theorem [7, (II.12)], the sum of the above ${ }_{3} \phi_{2}$ contains a factor $\left(q^{-k} ; q\right)_{n}$ which vanishes for $k<n$. Thus if $m<n$, then $\left\langle R_{m}, T_{n}\right\rangle_{\text {cher }}=0$.

When $k=n$ the the $q$-Pfaff-Saalschütz theorem [7, (II.12)] evaluates the above integral to

$$
\begin{aligned}
& \frac{1}{2 \pi i} \oint_{|z|=1}\left(t_{3} z, t_{3} / z ; q\right)_{n} T_{n}(z ; \mathbf{t} \mid q) w_{\text {cher }}(z ; \mathbf{t} \mid q) \frac{d z}{z} \\
& \quad=\frac{\left(t_{1} t_{2} t_{3} t_{4} q^{2 n} ; q\right)_{\infty}\left(-t_{1} t_{3}\right)^{n} q^{\left(\begin{array}{c}
n \\
2
\end{array}\right)}}{\left(q^{n+1}, q t_{1} t_{2}, t_{1} t_{3}, t_{1} t_{4}, t_{2} t_{3} q^{n}, t_{2} t_{4} q^{n}, t_{3} t_{4} q^{n} ; q\right)_{\infty}} .
\end{aligned}
$$

Using (2.9) an explicit computation shows that Proposition 3.4 holds for $m=n$.

Next we consider the case $m>n$. In this case consider the integral

$$
I=\frac{1}{2 \pi i} \oint_{|z|=1}\left(t_{1} z, q t_{1} / z ; q\right)_{j} R_{m}(z ; \mathbf{t} \mid q) w_{\text {cher }}(z ; \mathbf{t} \mid q) \frac{d z}{z} .
$$

After using (2.9) for $R_{m}, I$ is a constant multiple of

$$
\sum_{k=0}^{m} \frac{\left(q^{-m}, t_{1} t_{2} t_{3} t_{4} q^{m-1} ; q\right)_{k} q^{k}}{\left(q, t_{1} t_{3}, t_{2} t_{3}, t_{3} t_{4} ; q\right)_{k}} I_{j, k} .
$$

Applying Lemma 3.3 we see that the integral $I$ is a constant multiple of

$$
{ }_{3} \phi_{2}\left(\begin{array}{c}
q^{-m}, t_{1} t_{2} t_{3} t_{4} q^{m-1}, q^{j} t_{1} t_{3} \\
t_{1} t_{3}, t_{1} t_{2} t_{3} t_{4} q^{j}
\end{array} \mid q, q\right),
$$

which again by the $q$-Pfaff-Saalschütz theorem $\left[7,\left(\right.\right.$ II.12)] produces a factor $\left(q^{-j} ; q\right)_{m}$ and therefore vanishes for $j<m$.

Finally we come to the $T_{n}-T_{m}$ orthogonality, and finding the $L^{2}$-norms.

Proposition 3.5. We have the orthogonality relation

$$
\left\langle T_{m}, T_{n}\right\rangle_{\text {cher }}=\frac{\left(1-t_{1} t_{2}\right)\left(1-t_{3} t_{4} / q\right)}{\left(1-t_{1} t_{2} q^{n}\right)\left(1-t_{3} t_{4} q^{n-1}\right)} q^{n}\left\langle R_{m}, R_{n}\right\rangle_{\mathrm{cher}} \delta_{m, n} .
$$

Proof. We will use (2.4) and (2.10) to expand the T's, so consider the integral

$$
\begin{aligned}
& \frac{1}{2 \pi i} \oint_{|z|=1}\left(t_{1} z, q t_{1} / z ; q\right)_{j}\left(t_{2} z, q t_{2} / z ; q\right)_{k} w_{\text {cher }}(z ; \mathbf{t} \mid q) \frac{d z}{z} \\
& \quad=\frac{1}{2 \pi i} \oint_{|z|=1}\left(t_{1} z, t_{1} / z ; q\right)_{j}\left(t_{2} z, t_{2} / z ; q\right)_{k} \frac{\left(z-q^{k} t_{2}\right)\left(1-q^{j} t_{1} / z\right)}{z-1 / z} w_{\text {aw }}(z ; \mathbf{t} \mid q) \frac{d z}{z} \\
& \quad=\frac{\left(1-t_{1} t_{2} q^{k+j}\right)}{2 \pi} \int_{0}^{\pi} w_{\text {aw }}\left(\cos \theta ; t_{1} q^{j}, t_{2} q^{k}, t_{3}, t_{4} \mid q\right) d \theta \\
& \quad=\frac{\left(t_{1} t_{2} t_{3} t_{4} q^{k+j} ; q\right)_{\infty}}{\left(q, t_{1} t_{2} q^{j+k+1}, t_{1} t_{3} q^{j}, t_{1} t_{4} q^{j}, t_{2} t_{3} q^{k}, t_{2} t_{4} q^{k}, t_{3} t_{4} ; q\right)_{\infty}} .
\end{aligned}
$$

Using (2.4) to sum on $j$ we find

$$
\begin{aligned}
& \frac{1}{2 \pi i} \oint_{|z|=1} T_{n}(z ; \mathbf{t} \mid q)\left(t_{2} z, q t_{2} / z ; q\right)_{k} w_{\text {cher }}(z ; \mathbf{t} \mid q) \frac{d z}{z} \\
& \quad=\frac{\left(t_{1} t_{2} t_{3} t_{4} q^{k} ; q\right)_{\infty}}{\left(t_{1} t_{2} q^{k+1}, t_{1} t_{3}, t_{1} t_{4}, t_{2} t_{3} q^{k}, t_{2} t_{4} q^{k}, t_{3} t_{4} ; q\right)_{\infty}} 3 \phi_{2}\left(\begin{array}{c}
q^{-n}, t_{1} t_{2} t_{3} t_{4} q^{n-1}, t_{1} t_{2} q^{k+1} \\
q t_{1} t_{2}, t_{1} t_{2} t_{3} t_{4} q^{k}
\end{array} \mid q, q\right) .
\end{aligned}
$$


Again the $q$-Pfaff-Saalschütz theorem [7, (II.12)] evaluates the ${ }_{3} \phi_{2}$ as

$$
\frac{\left(q^{-k}, q^{2-n} / t_{3} t_{4} ; q\right)_{n}}{\left(q t_{1} t_{2}, q^{1-k-n} / t_{1} t_{2} t_{3} t_{4} ; q\right)_{n}},
$$

which vanishes when $k<n$. This establishes orthogonality for $m<n$, and the $k=n$ case establishes Proposition 3.5 for $m=n$ using (2.10).

To complete the proof of Theorem 3.1, we switch the orthogonality results for $R_{n}$ and $T_{n}$ to results for $R_{n}$ and $U_{n}$ using Proposition 2.1,

$$
c_{n} U_{n}=T_{n}-R_{n}, \quad c_{n}=\frac{q t_{1}\left(1-q^{-n}\right)\left(1-t_{1} t_{2} t_{3} t_{4} q^{n-1}\right)}{\left(1-t_{1} t_{2}\right)\left(1-q t_{1} t_{2}\right)\left(1-t_{1} t_{3}\right)\left(1-t_{1} t_{4}\right)} .
$$

The only remaining orthogonality relation we must check is

$$
\left\langle U_{n}, R_{n}\right\rangle_{\text {cher }}=c_{n}^{-1}\left\langle T_{n}-R_{n}, R_{n}\right\rangle_{\text {cher }}=c_{n}^{-1}\left(\left\langle T_{n}, R_{n}\right\rangle_{\text {cher }}-\left\langle R_{n}, R_{n}\right\rangle_{\text {cher }}\right)=0 .
$$

The value of $\left\langle U_{n}, U_{n}\right\rangle_{\text {cher }}$, which is non-zero, is found using Proposition 3.5,

$$
\begin{aligned}
\left\langle U_{n}, U_{n}\right\rangle_{\text {cher }} & =c_{n}^{-2}\left\langle T_{n}-R_{n}, T_{n}-R_{n}\right\rangle_{\text {cher }}=c_{n}^{-2}\left(\left\langle T_{n}, T_{n}\right\rangle_{\text {cher }}-\left\langle R_{n}, R_{n}\right\rangle_{\text {cher }}\right) \\
& =c_{n}^{-2}\left(\frac{\left(1-t_{1} t_{2}\right)\left(1-t_{3} t_{4} / q\right)}{\left(1-t_{1} t_{2} q^{n}\right)\left(1-t_{3} t_{4} q^{n-1}\right)} q^{n}-1\right)\left\langle R_{n}, R_{n}\right\rangle \\
& =c_{n}^{-2} \frac{\left(1-q^{n}\right)\left(1-t_{1} t_{2} t_{3} t_{4} q^{n-1}\right)}{\left(1-t_{1} t_{2} q^{n}\right)\left(1-t_{3} t_{4} q^{n-1}\right)}\left\langle R_{n}, R_{n}\right\rangle_{\text {cher }} .
\end{aligned}
$$

\section{More orthogonal bases for $V_{n}$}

In this section we find additional orthogonal bases for $V_{n}$, see Theorems 4.1, 4.4 and 4.5. We also explicitly give three and four term recurrence relations for some of these bases, which are the analogues of the recurrence relation for orthogonal polynomials.

The first result of this section is

Theorem 4.1. The set $\left\{T_{0}, \ldots, T_{n}, S_{1}, \ldots, S_{n}\right\}$ is an orthogonal basis for $V_{n}$ with respect to the symmetric bilinear from $\langle,\rangle_{\text {cher }}$. Moreover, $\left\langle T_{m}, T_{m}\right\rangle_{\text {cher }}$ and $\left\langle S_{m}, S_{m}\right\rangle_{\text {cher }}$ are non-zero.

Proof. Proposition 3.5 gives orthogonality of the $T_{n}$ 's. By Proposition 2.1 there are linear dependencies amongst $\left\{R_{n}, U_{n}, T_{n}\right\}$, and $\left\{R_{n}, U_{n}, S_{n}\right\}$. So $\left\langle S_{m}, S_{m}\right\rangle,\left\langle T_{m}, T_{m}\right\rangle$, and $\left\langle S_{m}, T_{m}\right\rangle$ may all be changed to the appropriate linear combinations of $R_{n}$ 's and $T_{m}$ 's. The results are given in the next proposition.

Proposition 4.2. For all $n \geq 1$ and $m \geq 0$ we have the orthogonality relations

$$
\begin{aligned}
& \left\langle S_{n}, T_{m}\right\rangle_{\text {cher }}=0, \\
& t_{1}\left\langle S_{n}, R_{m}\right\rangle_{\text {cher }}=\left(1-t_{1} t_{3}\right)\left(1-t_{1} t_{4}\right)\left\langle R_{n}, R_{m}\right\rangle_{\text {cher }} .
\end{aligned}
$$

For all $n \geq 1$ and $m \geq 1$ we have the orthogonality relations

$$
\left\langle S_{n}, S_{m}\right\rangle_{\mathrm{cher}}=\frac{q^{n}\left(1-t_{1} t_{3}\right)^{2}\left(1-t_{1} t_{4}\right)^{2}\left(1-t_{1} t_{2}\right)\left(1-t_{3} t_{4} / q\right)}{\left(q^{n}-1\right)\left(1-t_{1} t_{2} t_{3} t_{4} q^{n-1}\right) t_{1}^{2}}\left\langle R_{n}, R_{m}\right\rangle_{\mathrm{cher}} .
$$

Proof. This follows by direct calculation using Propositions 2.1, 3.4 and 3.5. 
Corollary 4.3. If $n \geq 1$, then for any $f \in V_{n-1}$ we have

$$
\left\langle R_{n}, f\right\rangle_{\text {cher }}=\left\langle S_{n}, f\right\rangle_{\text {cher }}=\left\langle T_{n}, f\right\rangle_{\text {cher }}=\left\langle U_{n}, f\right\rangle_{\text {cher }}=0 .
$$

Using Corollary 4.3 one may explicitly give many orthogonal bases using the Askey-Wilson polynomials $R_{n}$. For example, for the basis

$$
\left\{R_{0}, \ldots, R_{n}, T_{1}, \ldots, T_{n}\right\}
$$

of $V_{n}$, the only pairwise non-orthogonalities involve $T_{m}$ and $R_{m}$. One can replace these two polynomials by orthogonal linear combinations $R_{m}+c_{m} T_{m}$ and $R_{m}+d_{m} T_{m}$. The values of the constants $c_{m}$ and $d_{m}$ yielding orthogonality can be chosen using Propositions 3.4 and 3.5. The non-symmetric Askey-Wilson polynomial of Section 6 are one way of accomplishing this.

Another such choice corresponds to applying the Gram-Schmidt process to the ordered basis $\left\{1, z^{-1}, z^{1}, \ldots, z^{-n}, z^{n}\right\}$ on $V_{n}$ with $\langle,\rangle_{\text {cher }}$.

Theorem 4.4. An orthogonal basis for $V_{n}$ is $\left\{X_{0}, X_{-1}, X_{1}, \ldots, X_{-n}, X_{n}\right\}$, where

$$
\begin{array}{ll}
X_{-n}=\left(1-t_{1} t_{2}\right)\left(1-t_{1} t_{3}\right)\left(1-t_{1} t_{4}\right) R_{n}-t_{1}\left(1-t_{1} t_{2} t_{3} t_{4} q^{n-1}\right) S_{n}, \quad n>0, \\
X_{n}=\left(1-t_{1} t_{2}\right)\left(1-t_{1} t_{3}\right)\left(1-t_{1} t_{4}\right) R_{n}+t_{1}^{2} t_{2}\left(1-q^{n}\right) S_{n}, \quad n \geq 0 .
\end{array}
$$

Proof. The choice of the coefficients for $X_{-n}$ allows $X_{-n} \in V_{n-1} \oplus \operatorname{sp}\left\{z^{-n}\right\}$, while the coefficients for $X_{n}$ force $\left\langle X_{n}, X_{-n}\right\rangle_{\text {cher }}=0$ via Proposition 4.2. An explicit computation shows that all of the $L^{2}$-norms are non-zero.

For the ordered basis $\left\{1, z^{1}, z^{-1}, \ldots, z^{n}, z^{-n}\right\}$ on $V_{n}$ we have an analogous result.

Theorem 4.5. An orthogonal basis for $V_{n}$ is $\left\{Y_{0}, Y_{-1}, Y_{1}, \ldots, Y_{-n}, Y_{n}\right\}$, where

$$
\begin{aligned}
& Y_{-n}=q^{n-1} t_{3} t_{4}\left(1-t_{1} t_{2}\right)\left(1-t_{1} t_{3}\right)\left(1-t_{1} t_{4}\right) R_{n}-t_{1}\left(1-t_{1} t_{2} t_{3} t_{4} q^{n-1}\right) S_{n}, \quad n>0, \\
& Y_{n}=q^{n}\left(1-t_{1} t_{2}\right)\left(1-t_{1} t_{3}\right)\left(1-t_{1} t_{4}\right) R_{n}+t_{1}\left(1-q^{n}\right) S_{n}, \quad n \geq 0 .
\end{aligned}
$$

Orthogonal bases from Theorems 4.4 and 4.5 have a three term recurrence relation.

Proposition 4.6. For $n \geq 1$, there exist constants $a_{n}, b_{n}$ and $c_{n}$ such that

$$
z X_{-n}(z)=a_{n} X_{-n}(z)+b_{n} X_{n}(z)+c_{n} X_{n-1}(z)
$$

Proof. Because $X_{-n}$ has no $z^{n}$ term, $z X_{-n}(z) \in V_{n}$ and has an expansion

$$
z X_{-n}(z)=c_{0} X_{0}(z)=\sum_{i=1}^{n}\left(c_{i} X_{i}(z)+c_{-i} X_{-i}(z)\right) .
$$

By orthogonality, we can find the coefficients

$$
c_{k}=\frac{\left\langle z X_{-n}, X_{k}\right\rangle_{\text {cher }}}{\left\langle X_{k}, X_{k}\right\rangle_{\text {cher }}}=\frac{\left\langle X_{-n}, z X_{k}\right\rangle_{\text {cher }}}{\left\langle X_{k}, X_{k}\right\rangle_{\text {cher }}},
$$

as long as $\left\langle X_{k}, X_{k}\right\rangle_{\text {cher }} \neq 0$ (which is true here). The only terms to survive the orthogonality are $X_{n}, X_{-n}$, and $X_{n-1}$. The term $X_{1-n}$ does not survive because $z X_{1-n} \in V_{n-1}$ :

$$
\left\langle z X_{-n}, X_{1-n}\right\rangle_{\mathrm{cher}}=\left\langle X_{-n}, z X_{1-n}\right\rangle_{\mathrm{cher}}=0 .
$$

We record two propositions for these recurrences. The coefficients may be found by considering specific powers of $z$ on each side, we do not give the details of the computation. 
Proposition 4.7. The constants in Proposition 4.6 are

$$
\begin{aligned}
& a_{n}=\frac{\left(-t_{1}-t_{2}+t_{1} t_{2}\left(t_{3}+t_{4}\right) q^{n-1}\right)\left(1-t_{1} t_{2} t_{3} t_{4} q^{2 n-1}\right)}{\left(-1-t_{1} t_{2}+t_{1} t_{2} t_{3} t_{4} q^{n-1}+t_{1} t_{2} q^{n}\right)\left(1-t_{1} t_{2} t_{3} t_{4} q^{2 n-2}\right)} \\
& b_{n}=-\frac{\left(-t_{1}-t_{2}+t_{1} t_{2}\left(t_{3}+t_{4}\right) q^{n-1}\right)\left(1-t_{1} t_{2} t_{3} t_{4} q^{n-1}\right)\left(1-t_{3} t_{4} q^{n-1}\right)}{\left(-1-t_{1} t_{2}+t_{1} t_{2} t_{3} t_{4} q^{n-1}+t_{1} t_{2} q^{n}\right)\left(1-t_{1} t_{2} t_{3} t_{4} q^{2 n-2}\right)} \\
& c_{n}=\frac{t_{1}\left(1-t_{3} t_{4} q^{n-1}\right)\left(1-t_{2} t_{4} q^{n-1}\right)\left(1-t_{2} t_{3} q^{n-1}\right)}{\left(1-t_{1} t_{2} q^{n-1}\right)\left(1-t_{1} t_{2} t_{3} t_{4} q^{2 n-2}\right)}
\end{aligned}
$$

Proposition 4.8. For $n \geq 1$ we have

$$
\begin{aligned}
& \frac{1}{z} X_{n}=a_{n} X_{-n-1}+b_{n} X_{n}+c_{n} X_{-n}, \\
& a_{n}=\frac{\left(1-t_{1} t_{2} q^{n}\right)\left(1-t_{1} t_{3} q^{n}\right)\left(1-t_{1} t_{4} q^{n}\right)}{t_{1}\left(1-t_{3} t_{4} q^{n}\right)\left(1-t_{1} t_{2} t_{3} t_{4} q^{2 n}\right)}, \\
& b_{n}=\frac{\left(-t_{1}-t_{2}+t_{1} t_{2}\left(t_{3}+t_{4}\right) q^{n}\right)\left(1-t_{1} t_{2} t_{3} t_{4} q^{2 n-1}\right)}{\left(-1-t_{1} t_{2}+t_{1} t_{2} t_{3} t_{4} q^{n-1}+t_{1} t_{2} q^{n}\right)\left(1-t_{1} t_{2} t_{3} t_{4} q^{2 n}\right)}, \\
& c_{n}=-\frac{\left(1-q^{n}\right)\left(1-t_{1} t_{2} q^{n}\right)\left(-t_{1}-t_{2}+t_{1} t_{2}\left(t_{3}+t_{4}\right) q^{n}\right)}{\left(-1-t_{1} t_{2}+t_{1} t_{2} t_{3} t_{4} q^{n-1}+t_{1} t_{2} q^{n}\right)\left(1-t_{1} t_{2} t_{3} t_{4} q^{2 n}\right)} .
\end{aligned}
$$

There are four term relations for $z X_{n}$ and $\frac{1}{z} X_{-n}$.

Proposition 4.9. For $n \geq 1$, there exist constants $a_{n}, b_{n}, c_{n}$ and $d_{n}$ such that

$$
z X_{n}(z)=a_{n} Y_{-n-1}(z)+b_{n} X_{n}(z)+c_{n} Y_{-n}(z)+d_{n} X_{n-1}(z) .
$$

Proof. By orthogonality we have

$$
z X_{n}(z)=a_{n}^{\prime} X_{-n-1}(z)+b_{n}^{\prime} X_{n+1}(z)+c_{n}^{\prime} X_{-n}(z)+d_{n}^{\prime} X_{n}(z)+e_{n}^{\prime} X_{n-1}(z) .
$$

The sixth term $X_{1-n}(z)$ does not appear because $z X_{1-n} \in V_{n-1}$ :

$$
\left\langle z X_{n}, X_{1-n}\right\rangle_{\text {cher }}=\left\langle X_{n}, z X_{1-n}\right\rangle_{\text {cher }}=0 .
$$

Since $\operatorname{span}\left\{X_{-n-1}, X_{n+1}\right\}=\operatorname{span}\left\{Y_{-n-1}, Y_{n+1}\right\}$, the first two terms may be written as linear combination of $Y_{-n-1}$ and $Y_{n+1}$, But $z X_{-n}(z)$ has no $z^{-n-1}$ term, thus only $Y_{-n-1}$ appears. Because $\operatorname{span}\left\{X_{-n}, X_{n}\right\}=\operatorname{span}\left\{Y_{-n}, X_{n}\right\}$ we can replace $X_{n}$ by $Y_{-n}$.

Proposition 4.10. The constants in Proposition 4.9 are

$$
\begin{aligned}
& a_{n}=\frac{\left(1-t_{1} t_{2} q^{n}\right)\left(1-t_{1} t_{3} q^{n}\right)\left(1-t_{1} t_{4} q^{n}\right)\left(-1-t_{1} t_{2}+t_{1} t_{2} t_{3} t_{4} q^{n-1}+t_{1} t_{2} q^{n}\right)}{t_{1}\left(1-t_{3} t_{4} q^{n}\right)\left(1-t_{1} t_{2} t_{3} t_{4} q^{2 n-1}\right)\left(1-t_{1} t_{2} t_{3} t_{4} q^{2 n}\right)}, \\
& b_{n}=\frac{q^{n}\left(-1-t_{1} t_{2}+t_{1} t_{2} t_{3} t_{4} q^{n-1}+t_{1} t_{2} q^{n}\right)\left(-t_{3}-t_{4}+t_{2} t_{3} t_{4} q^{n}+t_{1} t_{3} t_{4} q^{n}\right)}{\left(1-t_{1} t_{2} t_{3} t_{4} q^{2 n-1}\right)\left(1-t_{1} t_{2} t_{3} t_{4} q^{2 n}\right)}, \\
& c_{n}=-\frac{\left(1-q^{n}\right)\left(1-t_{1} t_{2} q^{n}\right)\left(-t_{1}-t_{2}+t_{1} t_{2}\left(t_{3}+t_{4}\right) q^{n-1}\right)}{\left(1-t_{1} t_{2} t_{3} t_{4} q^{2 n-2}\right)\left(1-t_{1} t_{2} t_{3} t_{4} q^{2 n-1}\right)}, \\
& d_{n}=\frac{t_{1}\left(1-q^{n}\right)\left(1-t_{1} t_{2} q^{n}\right)\left(1-t_{2} t_{3} q^{n-1}\right)\left(1-t_{2} t_{4} q^{n-1}\right)\left(1-t_{3} t_{4} q^{n-1}\right)}{\left(1-t_{1} t_{2} q^{n-1}\right)\left(1-t_{1} t_{2} t_{3} t_{4} q^{2 n-2}\right)\left(1-t_{1} t_{2} t_{3} t_{4} q^{2 n-1}\right)} .
\end{aligned}
$$


Proposition 4.11. For $n \geq 1$ we have

$$
\begin{aligned}
& \frac{1}{z} X_{-n}=a_{n} X_{-n-1}+b_{n} X_{-n}+c_{n} Y_{n}+d_{n} Y_{n-1}, \\
& a_{n}=\frac{\left(1-t_{1} t_{2} q^{n}\right)\left(1-t_{1} t_{3} q^{n}\right)\left(1-t_{1} t_{4} q^{n}\right)\left(1-t_{3} t_{4} q^{n-1}\right)\left(1-t_{1} t_{2} t_{3} t_{4} q^{n-1}\right)}{t_{1}\left(1-t_{3} t_{4} q^{n}\right)\left(1-t_{1} t_{2} t_{3} t_{4} q^{2 n-1}\right)\left(1-t_{1} t_{2} t_{3} t_{4} q^{2 n}\right)}, \\
& b_{n}=\frac{q^{n-1}\left(-t_{3}-t_{4}+\left(t_{1}+t_{2}\right) t_{3} t_{4} q^{n-1}\right)\left(-1-t_{1} t_{2}+t_{1} t_{2} t_{3} t_{4} q^{n-1}+t_{1} t_{2} q^{n}\right)}{\left(1-t_{1} t_{2} t_{3} t_{4} q^{2 n-2}\right)\left(1-t_{1} t_{2} t_{3} t_{4} q^{2 n-1}\right)}, \\
& c_{n}=-\frac{\left(1-t_{3} t_{4} q^{n-1}\right)\left(1-t_{1} t_{2} t_{3} t_{4} q^{n-1}\right)\left(-t_{1}-t_{2}+t_{1} t_{2}\left(t_{3}+t_{4}\right) q^{n}\right)}{\left(1-t_{1} t_{2} t_{3} t_{4} q^{2 n-1}\right)\left(1-t_{1} t_{2} t_{3} t_{4} q^{2 n}\right)}, \\
& d_{n}=\frac{t_{1}\left(1-t_{2} t_{3} q^{n-1}\right)\left(1-t_{2} t_{4} q^{n-1}\right)\left(1-t_{3} t_{4} q^{n-1}\right)\left(-1-t_{1} t_{2}+t_{1} t_{2} t_{3} t_{4} q^{n-1}+t_{1} t_{2} q^{n}\right)}{\left(1-t_{1} t_{2} q^{n-1}\right)\left(1-t_{1} t_{2} t_{3} t_{4} q^{2 n-2}\right)\left(1-t_{1} t_{2} t_{3} t_{4} q^{2 n-1}\right)} .
\end{aligned}
$$

The two bases of Theorems 4.4 and 4.5 can be related, using (2.11). To explicitly show the parameter dependence we write $X_{n}(z ; \mathbf{t} \mid q)$ for $X_{n}$ and $Y_{n}(z ; \mathbf{t} \mid q)$ for $Y_{n}$.

Proposition 4.12. If $n \geq 1$, we have

$$
\begin{aligned}
& X_{-n}(1 / z ; 1 / \mathbf{t} \mid 1 / q)=-Y_{-n}(z ; \mathbf{t} \mid q) /\left(t_{1}^{3} t_{2} t_{3}^{2} t_{4}^{2} q^{n-1}\right), \\
& X_{n}(1 / z ; 1 / \mathbf{t} \mid 1 / q)=-Y_{n}(z ; \mathbf{t} \mid q) /\left(t_{1}^{3} t_{2} t_{3} t_{4} q^{n}\right) .
\end{aligned}
$$

Thus three term relations can be explicitly given for $z Y_{n}$ and $\frac{1}{z} Y_{-n}$, and four term relations for $z Y_{-n}$ and $\frac{1}{z} Y_{n}$.

\section{The operators $A_{0}$ and $A_{1}$}

Motivated by operators previously given in Noumi-Stokman [17], in this section we define linear transformations $A_{0}$ and $A_{1}$ on $V_{n}$. We explicitly find their actions on the possible bases $R_{m}$, $S_{m}, T_{m}$, and $U_{m}$. We use the self-adjointness of these operators to give an alternative proof of Theorems 3.1, 4.1, 4.4, and 4.5. We describe the eigenvalue problem whose solutions are the non-symmetric Askey-Wilson polynomials. We explicitly give the creation operators of NoumiStokman [17] via the three and four term recurrence relations of Section 4 . These results are in [17], but we include them for completeness.

For a Laurent polynomial $f \in V_{n}$, define

$$
\left(A_{0} f\right)(z)=\frac{\left(1-t_{3} / z\right)\left(1-t_{4} / z\right)}{\left(1-q / z^{2}\right)}(f(q / z)-f(z)),
$$

and

$$
\left(A_{1} f\right)(z)=\frac{\left(1-t_{1} z\right)\left(1-t_{2} z\right)}{\left(1-z^{2}\right)}(f(1 / z)-f(z)) .
$$

By a direct computation it is easy to see that each $A_{i}$ is a linear transformation from $V_{n}$ to $V_{n}$.

Proposition 5.1. Each $A_{i}: V_{n} \rightarrow V_{n}$ is self-adjoint, that is for any $f, g \in V_{n}$, we have

$$
\left\langle A_{0} f, g\right\rangle_{\text {cher }}=\left\langle f, A_{0} g\right\rangle_{\text {cher }}, \quad\left\langle A_{1} f, g\right\rangle_{\text {cher }}=\left\langle f, A_{1} g\right\rangle_{\text {cher }} .
$$


Proof. It is clear that $\left\langle A_{0} f, g\right\rangle_{\text {cher }}$ equals

$$
\begin{aligned}
\frac{1}{2 \pi i} \oint_{|z|=1} & \frac{\left(q, z^{2}, 1 / z^{2} ; q\right)_{\infty}}{\prod_{j=1}^{4}\left(t_{j} z, t_{j} / z ; q\right)_{\infty}} \frac{\left(1-t_{1} / z\right)\left(1-t_{2} / z\right)}{1-z^{-2}} \\
& \times \frac{\left(1-t_{3} / z\right)\left(1-t_{4} / z\right)}{\left(1-q / z^{2}\right)}[f(q / z) g(z)-f(z) g(z)] \frac{d z}{z} \\
= & \frac{1}{2 \pi i} \oint_{|z|=1} \frac{\left(q, z^{2}, q^{2} / z^{2} ; q\right)_{\infty}}{\prod_{j=1}^{4}\left(t_{j} z, q t_{j} / z ; q\right)_{\infty}}[f(q / z) g(z)-f(z) g(z)] \frac{d z}{z}
\end{aligned}
$$

This a difference of two integrals. In the first we let $z \rightarrow q / z$. A calculation shows that the first integral becomes

$$
\frac{1}{2 \pi i} \oint_{|z|=q} \frac{\left(q, z^{2}, q^{2} / z^{2} ; q\right)_{\infty}}{\prod_{j=1}^{4}\left(t_{j} z, q t_{j} / z ; q\right)_{\infty}} f(z) g(q / z) \frac{d z}{z} .
$$

We now deform the above contour to the circular contour $|z|=1$ because the integrand has no poles between the contours since each $\left|t_{i}\right|<1$. The proof of $\left\langle A_{1} f, g\right\rangle_{\text {cher }}=\left\langle f, A_{1} g\right\rangle_{\text {cher }}$ is similar and will be omitted.

Note that in the proof of Proposition 5.1 we only used the fact that $f$ and $g$ are analytic in $q \leq|z| \leq 1 / q$.

Proposition 5.2. The action of the operators $A_{0}$ and $A_{1}$ on the functions $R_{n}, T_{n}, S_{n}$, and $U_{n}$ is given by

$$
\begin{aligned}
& A_{0}\left(R_{n}\right)=\frac{t_{1}\left(q^{-n}-1\right)\left(1-t_{1} t_{2} t_{3} t_{4} q^{n-1}\right)}{\left(1-t_{1} t_{2}\right)\left(1-t_{1} t_{3}\right)\left(1-t_{1} t_{4}\right)} S_{n}=\alpha_{n} S_{n}, \quad A_{1}\left(R_{n}\right)=0 \\
& A_{0}\left(T_{n}\right)=0, \quad A_{1}\left(T_{n}\right)=\frac{q t_{1}\left(q^{-n}-1\right)\left(1-t_{1} t_{2} t_{3} t_{4} q^{n-1}\right)}{\left(1-q t_{1} t_{2}\right)\left(1-t_{1} t_{3}\right)\left(1-t_{1} t_{4}\right)} U_{n}=\beta_{n} U_{n} \\
& A_{0}\left(S_{n}\right)=\left(-1+t_{3} t_{4} / q\right) S_{n}, \quad A_{1}\left(S_{n}\right)=\frac{q^{1-n}\left(1-t_{1} t_{2} q^{n}\right)\left(1-t_{3} t_{4} q^{n-1}\right)}{\left(1-q t_{1} t_{2}\right)} U_{n}, \\
& A_{0}\left(U_{n}\right)=q^{-1}\left(1-q t_{1} t_{2}\right) S_{n}, \quad A_{1}\left(U_{n}\right)=\left(-1+t_{1} t_{2}\right) U_{n},
\end{aligned}
$$

respectively.

Proof. These follow by direct term by term application of $A_{0}$ and $A_{1}$ to the defining series.

These relations give another proof of the orthogonality relations in Theorems 3.1 and 4.1, for example

$$
\begin{aligned}
& 0=\left\langle A_{0}\left(T_{n}\right), R_{m}\right\rangle_{\text {cher }}=\left\langle T_{n}, A_{0}\left(R_{m}\right)\right\rangle_{\text {cher }}=\alpha_{m}\left\langle T_{n}, S_{m}\right\rangle_{\text {cher }}, \\
& 0=\left\langle A_{1}\left(R_{n}\right), U_{m}\right\rangle_{\text {cher }}=\left\langle R_{n}, A_{1}\left(U_{m}\right)\right\rangle_{\text {cher }}=\beta_{m}\left\langle R_{n}, U_{m}\right\rangle_{\text {cher }}
\end{aligned}
$$

From Proposition 5.2 the operators $A_{0}$ and $A_{1}$ act on the 2-dimensional space $W_{n}$ spanned by $\left\{R_{n}, S_{n}, U_{n}, T_{n}\right\}$. An elementary computation yields the next two theorems.

Theorem 5.3. If $n \geq 1$, the eigenvalues of $\left(A_{1}-t_{1} t_{2} I\right)\left(A_{0}-q^{-1} t_{3} t_{4} I\right)$ on $W_{n}$ are $q^{-n}$ and $t_{1} t_{2} t_{3} t_{4} q^{n-1}$, with corresponding eigenvectors $X_{-n}(z ; \mathbf{t} \mid q)$ and $Y_{n}(z ; \mathbf{t} \mid q)$. 
Theorem 5.4. If $n \geq 1$, the eigenvalues of $\left(A_{0}-q^{-1} t_{3} t_{4} I\right)\left(A_{1}-t_{1} t_{2} I\right)$ on $W_{n}$ are $q^{-n}$ and $t_{1} t_{2} t_{3} t_{4} q^{n-1}$, with corresponding eigenvectors $Y_{-n}(z ; \mathbf{t} \mid q)$ and $X_{n}(z ; \mathbf{t} \mid q)$.

These two theorems give alternative proofs of Theorems 4.4 and 4.5. For example

$$
\begin{aligned}
q^{-n}\left\langle X_{-n}, X_{n}\right\rangle_{\text {cher }} & =\left\langle\left(A_{1}-t_{1} t_{2} I\right)\left(A_{0}-q^{-1} t_{3} t_{4} I\right) X_{-n}, X_{n}\right\rangle_{\text {cher }} \\
& =\left\langle X_{-n},\left(A_{0}-q^{-1} t_{3} t_{4} I\right)\left(A_{1}-t_{1} t_{2} I\right) X_{n}\right\rangle_{\text {cher }} \\
& =t_{1} t_{2} t_{3} t_{4} q^{n-1}\left\langle X_{-n}, X_{n}\right\rangle_{\text {cher }}
\end{aligned}
$$

implying that $0=\left\langle X_{-n}, X_{n}\right\rangle_{\text {cher }}$.

Our next goal is to define ladder maps $\mathbb{S}_{0}$ and $\mathbb{S}_{1}$ such that $\mathbb{S}_{1}$ maps $Y_{n}$ to $X_{-n-1}$, and $\mathbb{S}_{0}$ maps $X_{-n}$ to $Y_{n}$. If such maps are found, then $Y_{n}$ is an $n^{\text {th }}$ iterate of their composition $\mathbb{S}_{0} \mathbb{S}_{1}$ applied to $Y_{0}$, see Theorems 5.9 and 5.12 .

Maps on $W_{n}$ that interchange the bases elements $\left\{X_{-n}, Y_{n}\right\}$ and $\left\{X_{n}, Y_{-n}\right\}$ can be defined using the commutators of $A_{i}$ with

$$
Y=\left(A_{1}-t_{1} t_{2} I\right)\left(A_{0}-q^{-1} t_{3} t_{4} I\right) \quad \text { and } \quad \mathbb{Y}=\left(A_{0}-q^{-1} t_{3} t_{4} I\right)\left(A_{1}-t_{1} t_{2} I\right) .
$$

From Proposition 5.2 the actions of $Y, \mathbb{Y}, A_{0}$ and $A_{1}$ are explicitly known on the 2-dimensional space $W_{n}=\operatorname{span}\left\{R_{n}, S_{n}, U_{n}, T_{n}\right\}$. An explicit computation yields the next two propositions.

Proposition 5.5. For $n \geq 1$ we have

$$
\begin{aligned}
& {\left[Y, A_{1}\right]\left(X_{-n}\right)=t_{1} t_{2} q^{-n}\left(1-t_{3} t_{4} q^{n-1}\right)\left(1-t_{1} t_{2} t_{3} t_{4} q^{n-1}\right) Y_{n},} \\
& {\left[\mathbb{Y}, A_{0}\right]\left(Y_{-n}\right)=-q^{-1} t_{3} t_{4}\left(1-t_{3} t_{4} q^{n-1}\right)\left(1-t_{1} t_{2} t_{3} t_{4} q^{n-1}\right) X_{n}}
\end{aligned}
$$

and if $n \geq 0$,

$$
\begin{aligned}
& {\left[Y, A_{1}\right]\left(Y_{n}\right)=q^{-n}\left(1-q^{n}\right)\left(1-t_{1} t_{2} q^{n}\right) X_{-n},} \\
& {\left[\mathbb{Y}, A_{0}\right]\left(X_{n}\right)=-q^{-2 n}\left(1-q^{n}\right)\left(1-t_{1} t_{2} q^{n}\right) Y_{-n} .}
\end{aligned}
$$

Proposition 5.6. For $n \geq 1$ we have

$$
\begin{aligned}
{\left[Y, A_{1}\right]\left(R_{n}\right)=} & \frac{t_{1} q^{1-2 n}\left(1-q^{n}\right)\left(1-t_{3} t_{4} q^{n-1}\right)\left(1-t_{1} t_{2} q^{n}\right)\left(1-t_{1} t_{2} t_{3} t_{4} q^{n-1}\right)}{\left(1-t_{1} t_{2}\right)\left(1-q t_{1} t_{2}\right) /\left(1-t_{1} t_{3}\right) /\left(1-t_{1} t_{4}\right)} U_{n} \\
{\left[Y, A_{1}\right]\left(U_{n}\right)=} & q^{-1} t_{2}\left(1-t_{1} t_{4}\right)\left(1-t_{1} t_{2}\right)\left(1-q t_{1} t_{2}\right)\left(1-t_{1} t_{3}\right) R_{n} \\
{\left[Y, A_{1}\right]\left(S_{n}\right)=} & -q^{-n} t_{2}\left(1-q^{n} t_{1} t_{2}\right)\left(1-t_{1} t_{3}\right)\left(1-t_{1} t_{4}\right)\left(1-t_{3} t_{4} q^{n-1}\right) R_{n} \\
& +q^{1-2 n} \frac{\left(1-q^{n}\right)\left(1-t_{1} t_{2} q^{n}\right)\left(1-t_{3} t_{4} q^{n-1}\right)\left(1-t_{1} t_{2} t_{3} t_{4} q^{n-1}\right)}{\left(1-t_{1} t_{2}\right)\left(1-q t_{1} t_{2}\right)} U_{n} \\
{\left[Y, A_{1}\right]\left(T_{n}\right)=} & -q^{-n} t_{1} t_{2}\left(1-t_{1} t_{2} t_{3} t_{4} q^{n-1}\right) R_{n} \\
& +q^{1-2 n} t_{1} \frac{\left(1-q^{n}\right)\left(1-t_{1} t_{2} q^{n}\right)\left(1-t_{3} t_{4} q^{n-1}\right)\left(1-t_{1} t_{2} t_{3} t_{4} q^{n-1}\right)}{\left(1-t_{1} t_{2}\right)\left(1-q t_{1} t_{2}\right)\left(1-t_{1} t_{3}\right)\left(1-t_{1} t_{4}\right)} U_{n} .
\end{aligned}
$$

We see from Proposition 5.5 that the commutator $\mathbb{S}_{0}=\left[Y, A_{1}\right]$ does map $X_{-n}$ to $Y_{n}$. To define an appropriate $\mathbb{S}_{1}$, we need another pair of operators.

We define operators $\hat{T}_{1}, \hat{T}_{2}: V_{n} \rightarrow V_{n+1}$ by

$$
\left(\hat{T}_{1} f\right)(z)=\frac{\left(A_{1} f\right)(z)+f(z)}{z}, \quad\left(\hat{T}_{2} f\right)(z)=z\left(\left(A_{0} f\right)(z)+f(z)\right) .
$$


Proposition 5.7. The action of $\hat{T}_{1}$ on $Y_{n}$ and $X_{-n}$ is given by

$$
\hat{T}_{1}\left(Y_{n}\right)=\frac{\left(1-q^{n} t_{1} t_{2}\right)\left(1-q^{n} t_{1} t_{3}\right)\left(1-q^{n} t_{1} t_{4}\right)}{t_{1}\left(1-q^{n} t_{3} t_{4}\right)\left(1-q^{2 n} t_{1} t_{2} t_{3} t_{4}\right)} X_{-n-1}+\frac{\left(t_{1}+t_{2}-q^{n} t_{1} t_{2}\left(t_{3}+t_{4}\right)\right)}{\left(1-q^{2 n} t_{1} t_{2} t_{3} t_{4}\right)} Y_{n}
$$

if $n \geq 0$ and

$$
\begin{aligned}
\hat{T}_{1}\left(X_{-n}\right)= & q^{n-1} \frac{t_{1} t_{2}\left(-t_{4}+t_{3}\left(-1+q^{n-1}\left(t_{1}+t_{2}\right) t_{4}\right)\right)}{\left(-1+q^{2(n-1)} t_{1} t_{2} t_{3} t_{4}\right)} X_{-n} \\
& +\frac{t_{1}^{2} t_{2}\left(1-q^{n-1} t_{2} t_{3}\right)\left(1-q^{n-1} t_{2} t_{4}\right)\left(1-q^{n-1} t_{3} t_{4}\right)}{\left(1-q^{n-1} t_{1} t_{2}\right)\left(-1+q^{2(n-1)} t_{1} t_{2} t_{3} t_{4}\right)} Y_{n-1},
\end{aligned}
$$

if $n \geq 1$, respectively.

Proof (sketch). Note that $\hat{T}_{1}=\frac{1}{z}\left(A_{1}+I\right)$. The action of $A_{1}$ on any element of $W_{n}$ is given by Proposition 5.2. The action of division by $z$ is given by Propositions 4.8, 4.11, and their $Y$ versions. Thus $\hat{T}_{1}\left(Y_{n}\right)$ and $\hat{T}_{1}\left(X_{-n}\right)$ may be given explicitly.

From Theorem 5.3 we know that $Y_{n}$ and $X_{-n}$ are eigenfunctions of $Y$, and can obtain Proposition 5.8 from Proposition 5.7.

Proposition 5.8. For $n \geq 0$ we have

$$
\begin{aligned}
& {\left[Y, \hat{T}_{1}\right]\left(Y_{n}\right)=-\frac{\left(1-t_{1} t_{2} q^{n}\right)\left(1-t_{1} t_{3} q^{n}\right)\left(1-t_{1} t_{4} q^{n}\right)}{t_{1} q^{n+1}\left(1-t_{3} t_{4} q^{n}\right)} X_{-n-1},} \\
& {\left[Y, \hat{T}_{1}\right]\left(X_{-n-1}\right)=-t_{1}^{2} t_{2} \frac{\left(1-t_{2} t_{3} q^{n}\right)\left(1-t_{2} t_{4} q^{n}\right)\left(1-t_{3} t_{4} q^{n}\right)}{q^{n+1}\left(1-t_{1} t_{2} q^{n}\right)} Y_{n} .}
\end{aligned}
$$

Finally we define $\mathbb{S}_{1}=\left[Y, \hat{T}_{1}\right]$, which maps $Y_{n}$ to $X_{-n-1}$. The next theorem is Proposition 9.3 in [17]. Recall that $Y_{0}=\left(1-t_{1} t_{2}\right)\left(1-t_{1} t_{3}\right)\left(1-t_{1} t_{4}\right)$ is a constant.

Theorem 5.9. We have

$$
c_{n} Y_{n}=\left(\mathbb{S}_{0} \mathbb{S}_{1}\right)^{n}\left(Y_{0}\right), \quad d_{n} X_{-n-1}=\mathbb{S}_{1}\left(\mathbb{S}_{0} \mathbb{S}_{1}\right)^{n}\left(Y_{0}\right)
$$

where

$$
\begin{aligned}
& c_{n}=\left(-t_{2}\right)^{n} q^{-n(n+1)}\left(t_{1} t_{2}, t_{1} t_{3}, t_{1} t_{4}, t_{1} t_{2} t_{3} t_{4} ; q\right)_{n}, \\
& d_{n}=-\left(-t_{2}\right)^{n} q^{-(n+1)^{2}}\left(t_{1} t_{2}, t_{1} t_{3}, t_{1} t_{4} ; q\right)_{n+1}\left(t_{1} t_{2} t_{3} t_{4} ; q\right)_{n} / t_{1}\left(1-t_{3} t_{4} q^{n}\right) .
\end{aligned}
$$

Completely analogous results hold for

$$
\hat{T}_{2}, \quad \mathbb{S}_{2}=\left[\mathbb{Y}, A_{0}\right], \quad \mathbb{S}_{3}=\left[\mathbb{Y}, \hat{T}_{2}\right],
$$

which we state below. Note that Proposition 5.5 shows that $\mathbb{S}_{2}$ interchanges $Y_{-n}$ and $X_{n}$.

Proposition 5.10. For $n \geq 0$ we have

$$
\begin{gathered}
\hat{T}_{2}\left(X_{n}\right)=\frac{t_{3}+t_{4}-t_{3} t_{4} q^{n}\left(t_{1}+t_{2}\right)}{\left(1-q^{2 n} t_{1} t_{2} t_{3} t_{4}\right)} X_{n}+\frac{\left(1-q^{n} t_{1} t_{2}\right)\left(1-q^{n} t_{1} t_{3}\right)\left(1-q^{n} t_{1} t_{4}\right)}{t_{1} q^{n}\left(1-q^{n} t_{3} t_{4}\right)\left(1-q^{2 n} t_{1} t_{2} t_{3} t_{4}\right)} Y_{-n-1}, \\
\hat{T}_{2}\left(Y_{-n-1}\right)=t_{1} t_{3} t_{4} q^{n} \frac{\left(1-q^{n} t_{2} t_{3}\right)\left(1-q^{n} t_{2} t_{4}\right)\left(1-q^{n} t_{3} t_{4}\right)}{\left(1-q^{n} t_{1} t_{2}\right)\left(1-q^{2 n} t_{1} t_{2} t_{3} t_{4}\right)} X_{-n} \\
+q^{n} t_{3} t_{4} \frac{\left(t_{2}+t_{1}-t_{1} t_{2} q^{n}\left(t_{3}+t_{4}\right)\right)}{\left(1-q^{2 n} t_{1} t_{2} t_{3} t_{4}\right)} Y_{-n-1} .
\end{gathered}
$$


The map $\mathbb{S}_{3}=\left[\mathbb{Y}, \hat{T}_{2}\right]$ interchanges $X_{n}$ and $Y_{-n-1}$.

Proposition 5.11. For $n \geq 0$ we have

$$
\begin{aligned}
& {\left[\mathbb{Y}, \hat{T}_{2}\right]\left(X_{n}\right)=\frac{\left(1-t_{1} t_{2} q^{n}\right)\left(1-t_{1} t_{3} q^{n}\right)\left(1-t_{1} t_{4} q^{n}\right)}{t_{1} q^{2 n+1}\left(1-t_{3} t_{4} q^{n}\right)} Y_{-n-1},} \\
& {\left[\mathbb{Y}, \hat{T}_{2}\right]\left(Y_{-n-1}\right)=q^{-1} t_{1} t_{3} t_{4} \frac{\left(1-t_{2} t_{3} q^{n}\right)\left(1-t_{2} t_{4} q^{n}\right)\left(1-t_{3} t_{4} q^{n}\right)}{\left(1-t_{1} t_{2} q^{n}\right)} X_{n} .}
\end{aligned}
$$

Recall that $X_{0}=\left(1-t_{1} t_{2}\right)\left(1-t_{1} t_{3}\right)\left(1-t_{1} t_{4}\right)$ is a constant.

Theorem 5.12. We have

$$
e_{n} X_{n}=\left(\mathbb{S}_{2} \mathbb{S}_{3}\right)^{n}\left(X_{0}\right), \quad f_{n} Y_{-n-1}=\mathbb{S}_{3}\left(\mathbb{S}_{2} \mathbb{S}_{3}\right)^{n}\left(X_{0}\right),
$$

where

$$
\begin{aligned}
& e_{n}=\left(-t_{3} t_{4} / t_{1}\right)^{n} q^{-n(n+1)}\left(t_{1} t_{2}, t_{1} t_{3}, t_{1} t_{4}, t_{1} t_{2} t_{3} t_{4} ; q\right)_{n}, \\
& f_{n}=\left(-t_{3} t_{4} / t_{1}\right)^{n} q^{-n(n+1)}\left(t_{1} t_{2}, t_{1} t_{3}, t_{1} t_{4} ; q\right)_{n+1}\left(t_{1} t_{2} t_{3} t_{4} ; q\right)_{n} / t_{1} q^{2 n+1}\left(1-t_{3} t_{4} q^{n}\right) .
\end{aligned}
$$

Since any two elements from $\left\{X_{n}, X_{-n}, Y_{n}, Y_{-n}\right\}$ form a basis for $W_{n}$, any element of $W_{n}$ has several such formulas.

\section{Non-symmetric Askey-Wilson polynomials}

The non-symmetric Askey-Wilson polynomials may be defined as the eigenfunctions given in Theorem 5.3, namely

$$
\left\{Y_{0}, Y_{1}, \ldots, Y_{n}, \ldots\right\} \cup\left\{X_{-1}, X_{-2}, \ldots, X_{-n}, \ldots\right\} .
$$

This set of Laurent polynomials also satisfy an biorthogonality relation which follows from Theorems 4.4 and 4.5 .

Define a new bilinear form on $V_{n}$ by

$$
\langle f, g\rangle_{\text {cher }^{\prime}}=\frac{1}{2 \pi i} \oint_{|z|=1} f(z) g(1 / z) w_{\text {cher }}(z ; \mathbf{t} \mid q) \frac{d z}{z} .
$$

Put

$$
P_{m}(z, \mathbf{t} \mid q)= \begin{cases}Y_{m}(z, \mathbf{t} \mid q) & \text { if } m \geq 0 \\ X_{m}(z, \mathbf{t} \mid q) & \text { if } m<0\end{cases}
$$

and $\left.P_{m}^{\prime}(z, \mathbf{t} \mid q)\right)=P_{m}(z, 1 / \mathbf{t} \mid 1 / q)$.

The biorthogonality relation with respect to this bilinear form [16], [17, $\S 6.7]$ is

Corollary 6.1. For $m \neq n,\left\langle P_{m}, P_{n}^{\prime}\right\rangle_{\text {cher }^{\prime}}=0$.

Proof. We use Proposition 4.12 to change the bilinear form $\langle,\rangle_{\text {cher }}$ to $\langle,\rangle_{\text {cher }}$. For example, if $m, n<0, m \neq n$, then

$$
\left\langle X_{m}, X_{n}^{\prime}\right\rangle_{\text {cher }^{\prime}}=-\left\langle X_{m}, Y_{n}\right\rangle_{\text {cher }} /\left(t_{1}^{3} t_{2} t_{3}^{2} t_{4}^{2} q^{n-1}\right)=0,
$$

while if $m<0$ and $n \geq 0$

$$
\left\langle X_{m}, Y_{n}^{\prime}\right\rangle_{\text {cher }}=-\left\langle X_{m}, X_{n}\right\rangle_{\text {cher }} t_{1}^{3} t_{2} t_{3} t_{4} q^{n}=0,
$$


The only term which requires checking is for $n>0$,

$$
\left\langle Y_{n}, X_{-n}^{\prime}\right\rangle_{\text {cher }^{\prime}}=-\left\langle Y_{n}, Y_{-n}\right\rangle_{\text {cher }} /\left(t_{1}^{3} t_{2} t_{3}^{2} t_{4}^{2} q^{n-1}\right)=0
$$

and

$$
\left\langle X_{-n}, Y_{n}^{\prime}\right\rangle_{\text {cher }^{\prime}}=-\left\langle X_{-n}, X_{n}\right\rangle_{\text {cher }} t_{1}^{3} t_{2} t_{3}^{2} t_{4}^{2} q^{n}=0
$$

by Theorems 4.4 and 4.5 .

The $L^{2}$-norms may also be found.

Corollary 6.2. We have for $n>0$,

$$
\begin{aligned}
\left\langle Y_{n}, Y_{n}^{\prime}\right\rangle_{\mathrm{cher}^{\prime}}= & -q^{n}\left\langle R_{n}, R_{n}\right\rangle_{\text {cher }} /\left(t_{1}^{3} t_{2} t_{3} t_{4}\right) \\
\times & \frac{\left(1-t_{1} t_{2}\right)\left(1-t_{1} t_{2} q^{n}\right)\left(1-t_{1} t_{3}\right)^{2}\left(1-t_{1} t_{4}\right)^{2}\left(1-t_{1} t_{2} t_{3} t_{4} q^{2 n-1}\right)}{\left(1-t_{1} t_{2} t_{3} t_{4} q^{n-1}\right)}, \\
\left\langle X_{-n}, X_{-n}^{\prime}\right\rangle_{\text {cher }^{\prime}}= & -t_{1}^{3} t_{2} t_{3}^{2} t_{4}^{2} q^{1-n}\left\langle R_{n}, R_{n}\right\rangle_{\text {cher }} \\
& \times \frac{\left(1-t_{1} t_{2}\right)\left(1-t_{3} t_{4} q^{n-1}\right)\left(1-t_{1} t_{3}\right)^{2}\left(1-t_{1} t_{4}\right)^{2}\left(1-t_{1} t_{2} t_{3} t_{4} q^{2 n-1}\right)}{\left(1-q^{n}\right)} .
\end{aligned}
$$

Proof. Using Proposition 4.12 we have

$$
\left\langle Y_{n}, Y_{n}^{\prime}\right\rangle_{\text {cher }^{\prime}}=-q^{n}\left\langle Y_{n}, X_{n}\right\rangle_{\text {cher }} /\left(t_{1}^{3} t_{2} t_{3} t_{4}\right),
$$

and $\left\langle Y_{n}, X_{n}\right\rangle_{\text {cher }}$ may be found using Theorems 4.4, 4.5 and Proposition 4.2. The proof for $\left\langle X_{-n}, X_{-n}^{\prime}\right\rangle_{\text {cher' }}$ is similar.

Koornwinder and Bouzeffour [13] gave a positive definite orthogonality relation for the nonsymmetric Askey-Wilson polynomials.

Macdonald [16, (6.6.8), p. 167] gives an expression for $P_{m}(z ; \mathbf{t} \mid q)$ as linear combination of $R_{m}(z ; \mathbf{t} \mid q)$ and $T_{m}(z ; \mathbf{t} \mid q)$. This is equivalent to the Noumi-Stokman expression using Proposition 2.1 .

Another $[17, \S 6.8]$ biorthogonal pair of bases with respect to $\langle,\rangle_{\text {cher }^{\prime}}$ is given by the bases

$$
\left\{R_{0}, \ldots, R_{n}, U_{1}, \ldots, U_{n}\right\} \quad \text { and } \quad\left\{R_{0}^{\prime}, \ldots, R_{n}^{\prime}, U_{1}^{\prime}, \ldots, U_{n}^{\prime}\right\} \text {. }
$$

The $U_{n}(z ; \mathbf{t} \mid q)$ are the anti-symmetric Askey-Wilson polynomials. However since

$$
R_{n}(1 / z ; 1 / \mathbf{t} \mid 1 / q)=R_{n}(z ; \mathbf{t} \mid q), \quad U_{n}(1 / z ; 1 / \mathbf{t} \mid 1 / q)=U_{n}(z ; \mathbf{t} \mid q) / t_{1} t_{2},
$$

this orthogonality is equivalent to Theorem 3.1.

\section{$7 \quad$ Askey-Wilson orthogonality}

One may ask if there are orthogonality relations for a basis of $V_{n}$ using the Askey-Wilson weight. By symmetry, one could take

$$
\left\{R_{0}, \ldots, R_{n},(z-1 / z),(z-1 / z) R_{1}, \ldots,(z-1 / z) R_{n-1}\right\} .
$$

We include a partial result in this direction. 
Theorem 7.1. We have the orthogonality relation

$$
\begin{aligned}
& \frac{1}{2 \pi i} \oint_{|z|=1} R_{m}(z ; \mathbf{t} \mid q) T_{n}(z ; \mathbf{t} \mid q) w_{a w}(x ; \mathbf{t} \mid q) \frac{d z}{z} \\
& =\frac{\left(-t_{1}^{2}\right)^{n} q^{\left(\begin{array}{c}
n \\
2
\end{array}\right)\left(1+q^{n}\right)\left(t_{1} t_{2} t_{3} t_{4} q^{2 n} ; q\right)_{\infty}}}{2 \prod_{1 \leq j<k \leq 4}\left(t_{j} t_{k} ; q\right)_{\infty}} \prod_{2 \leq j<k \leq 4}\left(t_{j} t_{k} ; q\right)_{n} \delta_{m, n},
\end{aligned}
$$

for $m \geq n$.

Proof. Consider the integral

$$
\frac{1}{2 \pi i} \oint_{|z|=1} R_{m}(z ; \mathbf{t} \mid q)\left(t_{1} z, q t_{1} / z ; q\right)_{k} w_{\mathrm{aw}}(x ; \mathbf{t} \mid q) \frac{d z}{z}
$$

for $0 \leq k \leq n$. The integral vanishes when $k=0$ by (2.1), so we assume $k>0$. Write $\left(t_{1} z, q t_{1} / z ; q\right)_{k}$ as $\left(1-t_{1} z\right)\left(1-t_{1} q^{k} / z\right)\left(q t_{1} z, q t_{1} / z ; q\right)_{k-1}$. Therefore the above integral is

$$
\frac{1}{2 \pi} \int_{-\pi}^{\pi} R_{m}\left(e^{i \theta} ; \mathbf{t} \mid q\right)\left(q t_{1} e^{i \theta}, q t_{1} e^{-i \theta} ; q\right)_{k-1}\left[1-t_{1}\left(1+q^{k}\right) \cos \theta+t_{1}^{2} q^{k}\right] w_{\mathrm{aw}}(x ; \mathbf{t} \mid q) d \theta,
$$

which vanishes for $k<m$ by (2.1). If $k=m$ the above integral is

$$
\begin{aligned}
& \frac{1+q^{n}}{4 \pi} \int_{-\pi}^{\pi} R_{m}\left(e^{i \theta} ; \mathbf{t} \mid q\right)\left(t_{1} e^{i \theta}, t_{1} e^{-i \theta} ; q\right)_{n} w_{\mathrm{aw}}(x ; \mathbf{t} \mid q) d \theta \\
& \quad=\frac{1+q^{n}}{4 \pi} \frac{\left(q, t_{1} t_{2}, t_{1}, t_{3}, t_{1} t_{4} ; q\right)_{n}}{q^{n}\left(q^{-n}, t_{1} t_{2} t_{3} t_{4} q^{n-1} ; q\right)_{n}} \int_{-\pi}^{\pi} R_{m}^{2}\left(e^{i \theta}\right) w_{\mathrm{aw}}(x ; \mathbf{t} \mid q) d \theta
\end{aligned}
$$

and the result follows from (1.1), (2.1) and (2.2).

\section{Recurrences}

In this section we record three term recurrence relations satisfied by $R_{n}, S_{n}, T_{n}$ and $U_{n}$. The three term recurrence relation for $R_{n}$ is the Askey-Wilson recurrence relation [12, (3.1.4)]

$$
\begin{aligned}
{[z+1 / z] R_{n}(z ; \mathbf{t} \mid q)=} & A_{n}^{(r)} R_{n+1}(z ; \mathbf{t} \mid q)+C_{n}^{(r)} R_{n-1}(z ; \mathbf{t} \mid q) \\
& +\left[-A_{n}^{(r)}-C_{n}^{(r)}+t_{1}+1 / t_{1}\right] R_{n}(z ; \mathbf{t} \mid q),
\end{aligned}
$$

where

$$
\begin{aligned}
A_{n}^{(r)}= & \frac{\left(1-t_{1} t_{2} t_{3} t_{4} q^{n-1}\right) \prod_{j=2}^{4}\left(1-t_{1} t_{j} q^{n}\right)}{t_{1}\left(1-t_{1} t_{2} t_{3} t_{4} q^{2 n-1}\right)\left(1-t_{1} t_{2} t_{3} t_{4} q^{2 n}\right)}, \\
C_{n}^{(r)}= & \frac{t_{1}\left(1-q^{n}\right) \prod_{2 \leq j<k \leq 4}\left(1-t_{j} t_{k} q^{n-1}\right)}{\left(1-t_{1} t_{2} t_{3} t_{4} q^{2 n-2}\right)\left(1-t_{1} t_{2} t_{3} t_{4} q^{2 n-1}\right)} .
\end{aligned}
$$

The three term recurrence relation for $T_{n}$ is

$$
\begin{aligned}
{[z+q / z] T_{n}(z ; \mathbf{t} \mid q)=} & A_{n}^{(t)} T_{n+1}(z ; \mathbf{t} \mid q)+C_{n}^{(t)} T_{n-1}(z ; \mathbf{t} \mid q) \\
& +\left[-A_{n}^{(t)}-C_{n}^{(t)}+q t_{1}+1 / t_{1}\right] T_{n}(z ; \mathbf{t} \mid q),
\end{aligned}
$$


with $n>0$, where

$$
\begin{aligned}
& A_{n}^{(t)}=\frac{\left(1-t_{1} t_{2} t_{3} t_{4} q^{n-1}\right)\left(1-t_{1} t_{2} q^{n+1}\right) \prod_{j=3}^{4}\left(1-t_{1} t_{j} q^{n}\right)}{t_{1}\left(1-t_{1} t_{2} t_{3} t_{4} q^{2 n-1}\right)\left(1-t_{1} t_{2} t_{3} t_{4} q^{2 n}\right)}, \\
& C_{n}^{(t)}=\frac{q t_{1}\left(1-q^{n}\right)\left(1-t_{3} t_{4} q^{n-2}\right) \prod_{j=3}^{4}\left(1-t_{2} t_{j} q^{n-1}\right)}{\left(1-t_{1} t_{2} t_{3} t_{4} q^{2 n-2}\right)\left(1-t_{1} t_{2} t_{3} t_{4} q^{2 n-1}\right)} .
\end{aligned}
$$

On the other hand the three term recurrence relation for $U_{n}$ is

$$
\begin{aligned}
{[z+q / z] U_{n}(z ; \mathbf{t} \mid q)=} & A_{n}^{(u)} U_{n+1}(z ; \mathbf{t} \mid q)+C_{n}^{(u)} U_{n-1}(z ; \mathbf{t} \mid q) \\
& +\left[-A_{n}^{(u)}-C_{n}^{(u)}+q t_{1}+q^{-1} / t_{1}\right] U_{n}(z ; \mathbf{t} \mid q),
\end{aligned}
$$

with $n>1$, where

$$
\begin{aligned}
A_{n}^{(u)} & =\frac{\left(1-t_{1} t_{2} t_{3} t_{4} q^{n}\right)\left(1-t_{1} t_{2} q^{n+1}\right) \prod_{j=3}^{4}\left(1-t_{1} t_{j} q^{n}\right)}{q t_{1}\left(1-t_{1} t_{2} t_{3} t_{4} q^{2 n-1}\right)\left(1-t_{1} t_{2} t_{3} t_{4} q^{2 n}\right)}, \\
C_{n}^{(u)} & =\frac{q t_{1}\left(1-q^{n-1}\right)\left(1-t_{1} t_{2} q^{n}\right)\left(1-t_{3} t_{4} q^{n-2}\right)}{\left(1-t_{1} t_{2} t_{3} t_{4} q^{2 n-2}\right)\left(1-t_{1} t_{2} t_{3} t_{4} q^{2 n-1}\right)} \prod_{j=3}^{4}\left(1-t_{1} t_{j} q^{n-1}\right)\left(1-t_{2} t_{j} q^{n-1}\right) .
\end{aligned}
$$

Finally the three term recurrence relation for $S_{n}$ is

$$
\begin{aligned}
{\left[q^{-1 / 2} z+q^{1 / 2} / z\right] R_{n}(z ; \mathbf{t} \mid q)=} & A_{n}^{(s)} R_{n+1}(z ; \mathbf{t} \mid q)+C_{n}^{(s)} R_{n-1}(z ; \mathbf{t} \mid q) \\
& +\left[-A_{n}^{(s)}-C_{n}^{(s)}+t_{1}+1 / t_{1}\right] R_{n}(z ; \mathbf{t} \mid q),
\end{aligned}
$$

and the coefficients are given by

$$
\begin{aligned}
& A_{n}^{(s)}= \frac{\left(1-t_{1} t_{2} t_{3} t_{4} q^{n}\right) \prod_{j=2}^{4}\left(1-t_{1} t_{j} q^{n}\right)}{t_{1}\left(1-t_{1} t_{2} t_{3} t_{4} q^{2 n-1}\right)\left(1-t_{1} t_{2} t_{3} t_{4} q^{2 n}\right)}, \\
& C_{n}^{(s)}=\frac{t_{1}\left(1-q^{n}\right) \prod_{2 \leq j<k \leq 4}\left(1-t_{j} t_{k} q^{n-1}\right)}{\left(1-t_{1} t_{2} t_{3} t_{4} q^{2 n-2}\right)\left(1-t_{1} t_{2} t_{3} t_{4} q^{2 n-1}\right)} .
\end{aligned}
$$

\section{$9 \quad$ Asymptotics}

Consider a general balanced terminating ${ }_{4} \phi_{3}$,

$$
{ }_{4} \phi_{3}\left(\begin{array}{c}
q^{-n}, A q^{n-1}, B, C \\
D, E, F
\end{array} \mid q, q\right), \quad \text { with } \quad A B C=D E F .
$$

Since the ${ }_{4} \phi_{3}$ is symmetric in $B$ and $C$ we may assume

$$
|B| \leq|C| \text {. }
$$

Ismail and Wilson [10] determined the large degree asymptotics of the Askey-Wilson polynomials. When $|B|<|C|$ their result is

$$
{ }_{4} \phi_{3}\left(\begin{array}{c}
q^{-n}, A q^{n-1}, B, C \\
D, E, F
\end{array} \mid q, q\right)=C^{n} \frac{(B, D / C, E / C, F / C ; q)_{\infty}}{(B / C, D, E, F ; q)_{\infty}}\left[1+\mathcal{O}\left(q^{n / 2}\right)\right] .
$$


On the other hand when $|B|=|C|$ we let $B / C=e^{2 i \theta}$ and in this case the Ismail-Wilson asymptotic result is

$$
\begin{aligned}
{ }_{4} \phi_{3}\left(\begin{array}{c}
q^{-n}, A q^{n-1}, B, C \\
D, E, F
\end{array} \mid q, q\right)=\left[1+\mathcal{O}\left(q^{n / 2}\right)\right] \\
\quad \times\left[\frac{C^{n}(B, D / C, E / C, F / C ; q)_{\infty}}{(B / C, D, E, F ; q)_{\infty}}+\frac{B^{n}(C, D / B, E / B, F / B ; q)_{\infty}}{(C / B, D, E, F ; q)_{\infty}}\right] .
\end{aligned}
$$

We now record the asymptotics of $R_{n}, S_{n}, T_{n}$ and $U_{n}$.

Theorem 9.1. For $|z|=1$ the following asymptotic formulas hold

$$
\begin{aligned}
& \prod_{j=2}^{4}\left(t_{1} t_{j} ; q\right)_{\infty} R_{n}(z ; \mathbf{t})=\left(\frac{t_{1}}{z}\right)^{n} \frac{\prod_{j=1}^{4}\left(t_{j} z ; q\right)_{\infty}}{\left(z^{2} ; q\right)_{\infty}}\left[1+\mathcal{O}\left(q^{n / 2}\right)\right] \\
& +\left(t_{1} z\right)^{n} \frac{\prod_{j=1}^{4}\left(t_{j} / z ; q\right)_{\infty}}{\left(1 / z^{2} ; q\right)_{\infty}}\left[1+\mathcal{O}\left(q^{n / 2}\right)\right], \\
& \frac{\left(q z^{-2} ; q\right)_{\infty} \prod_{j=2}^{4}\left(q t_{1} t_{j} ; q\right)_{\infty}}{\left(q t_{1} / z, q t_{2} / z, t_{3} / z, t_{4} / z ; q\right)_{\infty}} S_{n}(z ; \mathbf{t})=t_{1}^{n-1} z^{n}\left[1+\mathcal{O}\left(q^{n / 2}\right)\right] \\
& \frac{\left(q z^{-2} ; q\right)_{\infty}\left(q t_{1} t_{2} ; q\right)_{\infty} \prod_{j=3}^{4}\left(t_{1} t_{j} ; q\right)_{\infty}}{\prod_{j=1}^{2}\left(q t_{j} / z, t_{j+2} / z ; q\right)_{\infty}} T_{n}(z ; \mathbf{t})=\left(t_{1} z\right)^{n}\left[1+\mathcal{O}\left(q^{n / 2}\right)\right], \\
& \frac{\prod_{j=2}^{4}\left(q t_{1} t_{j} ; q\right)_{\infty}}{\left(1-q t_{1} t_{2}\right)} U_{n}(z ; \mathbf{t})=\frac{\left(q t_{1}\right)^{n-1}}{z^{n}} \frac{\prod_{j=1}^{4}\left(t_{j} z ; q\right)_{\infty}}{\left(z^{2} ; q\right)_{\infty}}\left[1+\mathcal{O}\left(q^{n / 2}\right)\right] \\
& +\left(q t_{1}\right)^{n-1} z^{n-2} \frac{\left(1-t_{1} z\right)\left(1-t_{2} z\right) \prod_{j=1}^{4}\left(t_{j} / z ; q\right)_{\infty}}{\left(1 / z^{2} ; q\right)_{\infty}\left(1-t_{1} / z\right)\left(1-t_{2} / z\right)}\left[1+\mathcal{O}\left(q^{n / 2}\right)\right] .
\end{aligned}
$$

\section{Discrete orthogonality}

The Askey-Wilson polynomials have a discrete orthogonality, the $q$-Racah orthogonality $[2,12]$, when $t_{1} t_{j}=q^{-N}$, for some $j=2,3$ or 4 , and for some positive integer $N$. In this section we give the corresponding discrete orthogonality for the Laurent polynomials. This may be done by deforming the contours, we do not give the details.

The $q$-Racah measure for the Askey-Wilson polynomials is purely discrete and based upon the very well poised terminating ${ }_{6} \phi_{5}$ evaluation [7, (II.20)]

$$
\begin{gathered}
\sum_{k=0}^{N} \frac{\left(t_{1}^{2}, t_{1} t_{2}, t_{1} t_{3}, t_{1} t_{4} ; q\right)_{k}}{\left(q, q t_{1} / t_{2}, q t_{1} / t_{3}, q t_{1} / t_{4} ; q\right)_{k}} \frac{1-t_{1}^{2} q^{2 k}}{1-t_{1}^{2}}\left(\frac{q}{t_{1} t_{2} t_{3} t_{4}}\right)^{k} \\
=\frac{\left(q t_{1}^{2}, q t_{1}^{2} / t_{2} t_{3}, q t_{1}^{2} / t_{2} t_{4}, q t_{1}^{2} / t_{3} t_{4} ; q\right)_{\infty}}{\left(q t_{1} / t_{2}, q t_{1} / t_{3}, q t_{1} / t_{4}, q / t_{1} t_{2} t_{3} t_{4} ; q\right)_{\infty}}
\end{gathered}
$$


Using

$$
\frac{1-t_{1}^{2} q^{2 k}}{1-t_{1}^{2}}=\frac{1}{\left(1-t_{1}^{2}\right)\left(1-t_{1} t_{2}\right)}\left(-t_{1} t_{2}\left(1-q^{k}\right)\left(1-q^{k} t_{1} / t_{2}\right)+\left(1-t_{1}^{2} q^{k}\right)\left(1-t_{1} t_{2} q^{k}\right)\right)
$$

one can rewrite the left side of the ${ }_{6} \phi_{5}$ evaluation (10.1) as a sum of two ${ }_{4} \phi_{3}$ 's

$$
\begin{gathered}
\sum_{k=1}^{N} \frac{\left(q t_{1}^{2}, q t_{1} t_{2} ; q\right)_{k-1}\left(t_{1} t_{3}, t_{1} t_{4} ; q\right)_{k}}{\left(q t_{1} / t_{2}, q ; q\right)_{k-1}\left(q t_{1} / t_{3}, q t_{1} / t_{4} ; q\right)_{k}}\left(\frac{q}{t_{1} t_{2} t_{3} t_{4}}\right)^{k}\left(-t_{1} t_{2}\right) \\
\quad+\sum_{k=0}^{N} \frac{\left(q t_{1}^{2}, q t_{1} t_{2}, t_{1} t_{3}, t_{1} t_{4} ; q\right)_{k}}{\left(q t_{1} / t_{2}, q t_{1} / t_{3}, q t_{1} / t_{4}, q ; q\right)_{k}}\left(\frac{q}{t_{1} t_{2} t_{3} t_{4}}\right)^{k} .
\end{gathered}
$$

Definition 10.1. If $t_{1} t_{j}=q^{-N}$ for some positive integer $N$, the Racah bilinear form on $V_{N}$ is defined by

$$
\begin{aligned}
\langle f, g\rangle_{\text {Racah }}= & \sum_{k=1}^{N} \frac{\left(q t_{1}^{2}, q t_{1} t_{2} ; q\right)_{k-1}\left(t_{1} t_{3}, t_{1} t_{4} ; q\right)_{k}}{\left(q t_{1} / t_{2}, q ; q\right)_{k-1}\left(q t_{1} / t_{3}, q t_{1} / t_{4} ; q\right)_{k}}\left(\frac{q}{t_{1} t_{2} t_{3} t_{4}}\right)^{k}\left(-t_{1} t_{2}\right) f\left(t_{1} q^{k}\right) g\left(t_{1} q^{k}\right) \\
& +\sum_{k=0}^{N} \frac{\left(q t_{1}^{2}, q t_{1} t_{2}, t_{1} t_{3}, t_{1} t_{4} ; q\right)_{k}}{\left(q t_{1} / t_{2}, q t_{1} / t_{3}, q t_{1} / t_{4}, q ; q\right)_{k}}\left(\frac{q}{t_{1} t_{2} t_{3} t_{4}}\right)^{k} f\left(q^{-k} / t_{1}\right) g\left(q^{-k} / t_{1}\right) .
\end{aligned}
$$

Theorem 10.2. If $t_{1} t_{j}=q^{-N}$ for some positive integer $N$ and $j=3$ or 4 , then

$$
\begin{aligned}
& \left\{R_{0}, \ldots, R_{N}, U_{1}, \ldots, U_{N}\right\}, \quad\left\{T_{0}, \ldots, T_{N}, S_{1}, \ldots, S_{N}\right\}, \\
& \left\{X_{-N}, \ldots, X_{0}, X_{1}, \ldots, X_{N}\right\} \quad \text { and } \quad\left\{Y_{-N}, \ldots, Y_{0}, Y_{1}, \ldots, Y_{N}\right\}
\end{aligned}
$$

are Racah-orthogonal bases for $V_{N}$.

If $t_{1} t_{2}=q^{-N}$, the Racah bilinear form contains a zero term: the $k=N$ term in the second sum is 0 due to $\left(q t_{1} t_{2} ; q\right)_{k}$. In this case $U_{N}$ and $T_{N}$ are not well-defined. A slight modification of Theorem 10.2 can be formulated, we do not give it here.

\section{Acknowledgements}

The research of Mourad E.H. Ismail is partially supported by Research Grants Council of Hong Kong under contracts \#101410 and \#101411 and by King Saud University, Riyadh through grant DSFP/MATH 01.

\section{References}

[1] Andrews G.E., Askey R., Roy R., Special functions, Encyclopedia of Mathematics and its Applications, Vol. 71, Cambridge University Press, Cambridge, 1999.

[2] Askey R., Wilson J., A set of orthogonal polynomials that generalize the Racah coefficients or $6-j$ symbols, SIAM J. Math. Anal. 10 (1979), 1008-1016.

[3] Askey R., Wilson J., Some basic hypergeometric orthogonal polynomials that generalize Jacobi polynomials, Mem. Amer. Math. Soc. 54 (1985), no. 319, iv+55 pages.

[4] Brezinski C., Biorthogonality and its applications to numerical analysis, Monographs and Textbooks in Pure and Applied Mathematics, Vol. 156, Marcel Dekker Inc., New York, 1992.

[5] Bultheel A., Gonzalez-Vera P., Hendriksen E., Njastad O., Orthogonal rational functions and continued fractions, in Special Functions 2000: Current Perspective and Future Directions (Tempe, AZ), NATO Sci. Ser. II Math. Phys. Chem., Vol. 30, Kluwer Acad. Publ., Dordrecht, 2001, 87-109. 
[6] Cherednik I., Nonsymmetric Macdonald polynomials, Int. Math. Res. Not. 1995 (1995), no. 10, 483-515, q-alg/9505029.

[7] Gasper G., Rahman M., Basic hypergeometric series, Encyclopedia of Mathematics and its Applications, Vol. 96, 2nd ed., Cambridge University Press, Cambridge, 2004.

[8] Ismail M.E.H., Classical and quantum orthogonal polynomials in one variable, Encyclopedia of Mathematics and its Applications, Vol. 98, Cambridge University Press, Cambridge, 2005.

[9] Ismail M.E.H., Masson D.R., Generalized orthogonality and continued fractions, J. Approx. Theory 83 (1995), 1-40, math.CA/9407213.

[10] Ismail M.E.H., Wilson J.A., Asymptotic and generating relations for the $q$-Jacobi and ${ }_{4} \varphi_{3}$ polynomials, J. Approx. Theory 36 (1982), 43-54.

[11] Jones W.B., Thron W.J., Continued fractions, Encyclopedia of Mathematics and its Applications, Vol. 11, Addison-Wesley Publishing Co., Reading, Mass., 1980.

[12] Koekoek R., Swarttouw R.F., The Askey-scheme of hypergeometric orthogonal polynomials and its $q$ analogue, Report 98-17, Faculty of Technical Mathematics and Informatics, Delft University of Technology, 1998, http://aw.twi.tudelft.nl/ koekoek/askey/.

[13] Koornwinder T.H., Bouzeffour F., Non-symmetric Askey-Wilson polynomials as vector-valued polynomials, Appl. Anal. 90 (2011), 731-746, arXiv:1006.1140.

[14] Lorentzen L., Waadeland H., Continued fractions with applications, Studies in Computational Mathematics, Vol. 3, North-Holland Publishing Co., Amsterdam, 1992.

[15] Lorentzen L., Waadeland H., Continued fractions, Vol. 1, Convergence theory, 2nd ed., Atlantis Studies in Mathematics for Engineering and Science, Atlantis Press, Paris, 2008.

[16] Macdonald I.G., Affine Hecke algebras and orthogonal polynomials, Cambridge Tracts in Mathematics, Vol. 157, Cambridge University Press, Cambridge, 2003.

[17] Noumi M., Stokman J.V., Askey-Wilson polynomials: an affine Hecke algebra approach, in Laredo Lectures on Orthogonal Polynomials and Special Functions, Adv. Theory Spec. Funct. Orthogonal Polynomials, Nova Sci. Publ., Hauppauge, NY, 2004, 111-144, math.QA/0001033.

[18] Spiridonov V., Zhedanov A., Spectral transformation chains and some new biorthogonal rational functions, Comm. Math. Phys. 210 (2000), 49-83.

[19] Wilkinson J.H., The algebraic eigenvalue problem, Clarendon Press, Oxford, 1965.

[20] Wilson J.A., Private communication, 1980.

[21] Zhedanov A., Biorthogonal rational functions and the generalized eigenvalue problem, J. Approx. Theory 101 (1999), 303-329. 